\title{
Multimodal transcranial magnetic stimulation: using concurrent neuroimaging to reveal the neural network dynamics of noninvasive brain stimulation
}

Citation for published version (APA):

Reithler, J., Peters, J. C., \& Sack, A. T. (2011). Multimodal transcranial magnetic stimulation: using concurrent neuroimaging to reveal the neural network dynamics of noninvasive brain stimulation. Progress in Neurobiology, 94(2), 149-165. https://doi.org/10.1016/j.pneurobio.2011.04.004

Document status and date:

Published: 01/01/2011

DOI:

10.1016/j.pneurobio.2011.04.004

Document Version:

Publisher's PDF, also known as Version of record

Document license:

Taverne

Please check the document version of this publication:

- A submitted manuscript is the version of the article upon submission and before peer-review. There can be important differences between the submitted version and the official published version of record.

People interested in the research are advised to contact the author for the final version of the publication, or visit the DOI to the publisher's website.

- The final author version and the galley proof are versions of the publication after peer review.

- The final published version features the final layout of the paper including the volume, issue and page numbers.

Link to publication

\footnotetext{
General rights rights.

- You may freely distribute the URL identifying the publication in the public portal. please follow below link for the End User Agreement:

www.umlib.nl/taverne-license

Take down policy

If you believe that this document breaches copyright please contact us at:

repository@maastrichtuniversity.nl

providing details and we will investigate your claim.
}

Copyright and moral rights for the publications made accessible in the public portal are retained by the authors and/or other copyright owners and it is a condition of accessing publications that users recognise and abide by the legal requirements associated with these

- Users may download and print one copy of any publication from the public portal for the purpose of private study or research.

- You may not further distribute the material or use it for any profit-making activity or commercial gain

If the publication is distributed under the terms of Article 25fa of the Dutch Copyright Act, indicated by the "Taverne" license above, 


\title{
Multimodal transcranial magnetic stimulation: Using concurrent neuroimaging to reveal the neural network dynamics of noninvasive brain stimulation
}

\author{
J. Reithler ${ }^{\mathrm{a}, \mathrm{b}, *}$, J.C. Peters ${ }^{\mathrm{a}, \mathrm{b}, \mathrm{c}, 1}$, A.T. Sack ${ }^{\mathrm{a}, \mathrm{b}, 2}$ \\ ${ }^{a}$ Cognitive Neuroscience Department, Faculty of Psychology \& Neuroscience, Maastricht University, Maastricht, The Netherlands \\ ${ }^{\mathrm{b}}$ Maastricht Brain Imaging Center (M-BIC), Maastricht, The Netherlands \\ ${ }^{\mathrm{c}}$ Netherlands Institute for Neuroscience, an Institute of the Royal Netherlands Academy for Arts and Sciences (KNAW), Amsterdam, The Netherlands
}

\section{A R T I C L E I N F O}

\section{Article history:}

Received 20 December 2010

Received in revised form 31 March 2011

Accepted 6 April 2011

Available online 16 April 2011

\section{Keywords:}

Transcranial magnetic stimulation (TMS)

Electroencephalography (EEG)

Positron emission tomography (PET)

Functional magnetic resonance imaging

(fMRI)

Multimodal imaging

Connectivity

\begin{abstract}
A B S T R A C T
Since its introduction in the 1980s, Transcranial Magnetic Stimulation (TMS) has proven to be a versatile method to non-invasively study human brain function by reversibly altering ongoing neural processing. In addition, TMS has been explored as a therapeutic intervention in a number of neurological and neuropsychiatric conditions. However, our understanding of TMS-induced changes in neural activity patterns is still rather limited, particularly when it comes to changes in neural network dynamics beyond the cortical site directly targeted by TMS. In order to monitor both its local and remote neurophysiological effects, TMS has been combined with complementary neuroimaging methods that allow additional insights into how observed TMS effects at the behavioral level can be interpreted by taking into account the full scale of its impact throughout the brain. The current review provides a comprehensive overview of the existing multimodal TMS literature, covering studies in which TMS was combined with one of the three main neuroimaging modalities, namely Electroencephalography, Positron Emission Tomography, and functional Magnetic Resonance Imaging. Besides constituting a reflection of the status quo in this exciting multidisciplinary research field, this review additionally reveals both convergent and divergent observations across modalities that await corroboration or resolution, thereby further guiding ongoing basic research and providing useful constraints to optimize future clinical applications.
\end{abstract}

(c) 2011 Elsevier Ltd. All rights reserved.

\section{Contents}

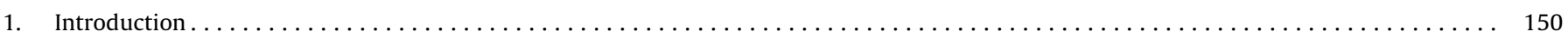

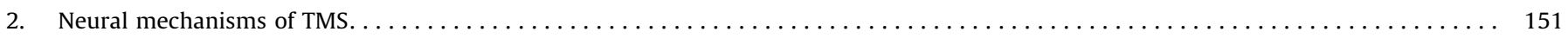

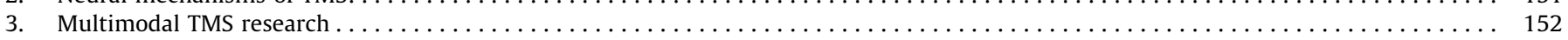

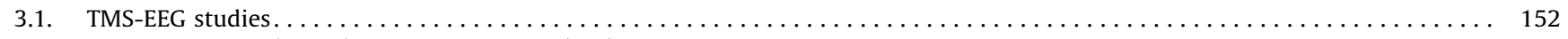

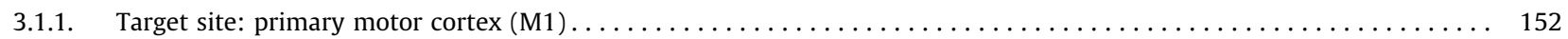

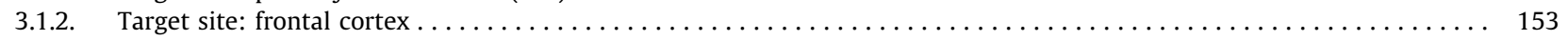

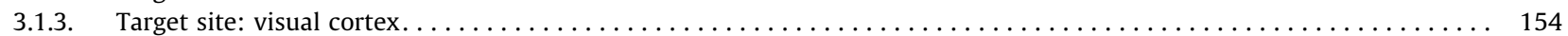

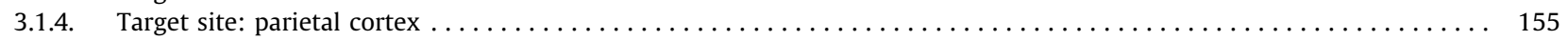

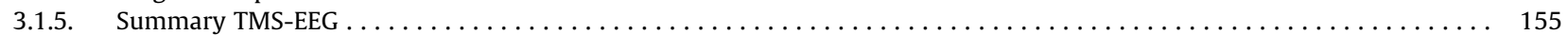

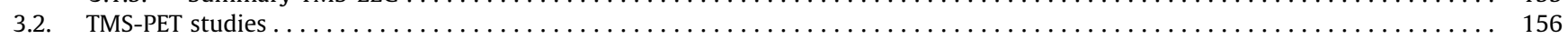

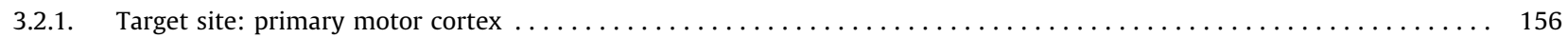

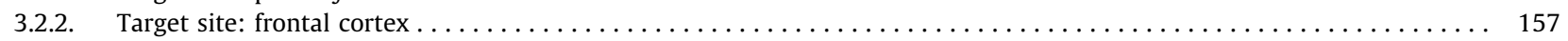

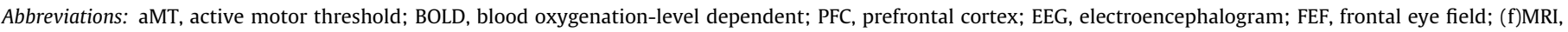

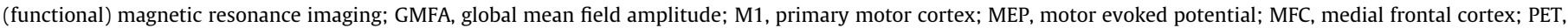

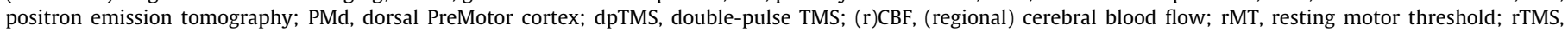

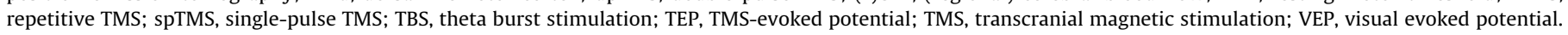

* Corresponding author at: Cognitive Neuroscience Department, Faculty of Psychology \& Neuroscience, Maastricht University, PO Box 616, 6200 MD Maastricht, the Netherlands. Tel.: +31 43 3881896; fax: +31 433884125.

E-mail addresses: j.reithler@maastrichtuniversity.nl (J. Reithler), j.peters@nin.knaw.nl (J.C. Peters), a.sack@maastrichtuniversity.nl (A.T. Sack).

1 Tel.: +31 20 5665104; fax: +31205666121.

2 Tel.: +31 43 3884267; fax: +31433884125.
} 


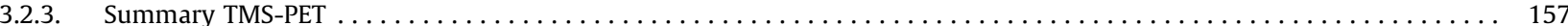

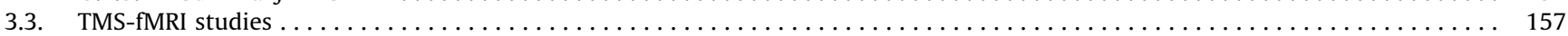

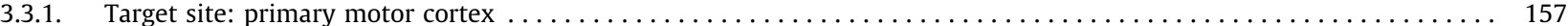

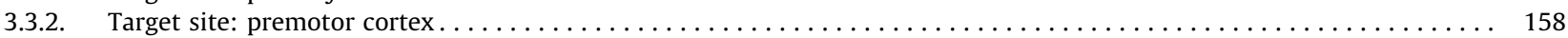

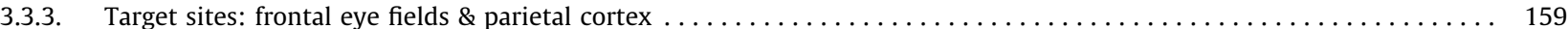

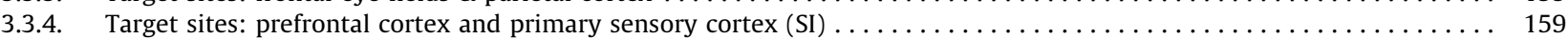

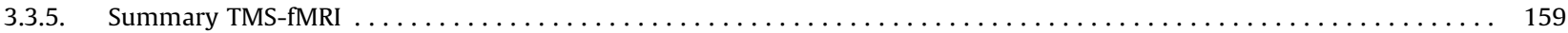

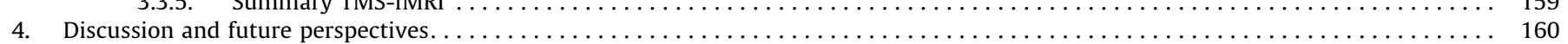

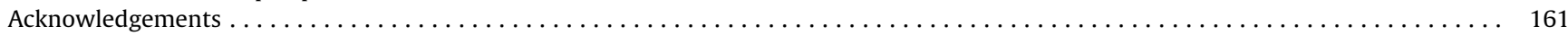

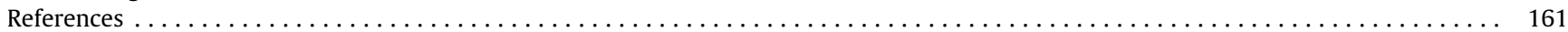

\section{Introduction}

Transcranial magnetic stimulation (TMS) is a versatile method which allows to non-invasively probe, and reversibly alter, neural processing in the working human brain (Barker et al., 1985; Wassermann et al., 2008). In brief, TMS involves a short-lasting (100-400 $\mu \mathrm{s})$ capacitor discharge of electric current into a stimulation coil, producing a magnetic field in the range of 1.5$2 \mathrm{~T}$. In turn, this change in magnetic field is accompanied by an electric field (up to $\sim 200 \mathrm{~V} / \mathrm{m}$ ) leading to depolarizing membrane potentials in the nearby cortical tissue under the coil, affecting ongoing neural activity patterns. Next to its potential therapeutic effect in a variety of neurological and psychiatric conditions (Kobayashi and Pascual-Leone, 2003; Miniussi et al., 2008; but see Ridding and Rothwell, 2007), TMS has become a particularly relevant research tool in the cognitive neurosciences (PascualLeone et al., 2000; Walsh and Cowey, 2000; Sack, 2006). One of the most commonly used applications of TMS is the so-called 'virtual lesion' approach, in which neural processing is temporarily disturbed to assess the causal relevance of the stimulated brain region in successfully completing the task at hand. Due to its reversibility and spatio-temporal precision, this approach allows collecting data on brain-behavior relations in a more controlled fashion than studies on naturally occurring brain lesions. In this way, TMS nicely complements neuropsychological work in patients, intracortical electrical microstimulation data obtained in animal models, and the correlational approaches commonly used in human neuroimaging studies. In addition, under certain circumstances, TMS can influence ongoing neural processing in such a way that it actually enhances cortical excitability or facilitates behavioral task performance, for example by influencing the mutual inhibition between areas (Hilgetag et al., 2001; Walsh et al., 1998; see also Reis et al., 2008). Or, alternatively, TMS can be used as a well-controlled probe of the brain's current state, charting changes in brain responses to an external stimulus (i.e., the TMS pulse, which for example can by-pass early sensory processing stages) depending on the participant's conscious state (Massimini et al., 2005) or looking at the effectiveness of signal transmission without affecting actual task performance (Morishima et al., 2009). All these applications emphasize the versatility of TMS, related to the many degrees of freedom that the TMS parameter space provides, in terms of possible combinations of stimulation intensity, frequency, duration, and cortical target site. Crucially, especially in light of potential clinical applications of TMS, the right combination of stimulation parameters can also have prolonged effects on neural activity beyond the period of stimulation itself. Such protocols require multiple repetitions of stimulation at a particular frequency, and are therefore called 'repetitive' (or in short, rTMS) protocols. The recently introduced Theta Burst Stimulation (TBS, Huang et al., 2005) is a prominent example of this class of protocols due to its effectiveness (only $40 \mathrm{~s}$ of stimulation can lead to after-effects lasting $\geq 1 \mathrm{~h}$ ).

When TMS is used to investigate the neurobiological basis of human cognition and behavior, behavioral responses (in terms of reaction time, accuracy, sensory detection or discrimination threshold, etc.) have been, and always will be, valuable outcome measures to index the effects of the applied TMS protocol. However, based on behavioral measures alone, it is virtually impossible to infer the full scale of neural network mechanisms affected by TMS. One way to partially overcome this drawback is to revert to elegant experimental designs involving two TMS coils over different target sites and asynchronies in the timing of TMS pulse delivery, providing interesting insights into the interplay between sets of distant brain regions (e.g., Davare et al., 2010). Nevertheless, to understand how underlying neural network processes are modulated, changes in brain activity induced by TMS should be monitored directly. By combining TMS with other neuroimaging techniques (referred to as 'multimodal imaging'), both the instant and more long-lasting neurophysiological consequences of applying TMS can be assessed (Sack and Linden, 2003; Siebner et al., 2009). Most importantly, the ability to monitor what happens in the rest of the brain while targeting a particular cortical site can reveal the full network dynamics reflecting the impact of TMS, instead of only assuming certain corollary activation changes or ignoring potential remote effects of TMS altogether.

The purpose of the current review is to provide a brief and accessible, yet comprehensive overview of the existing neuroscientific literature on the combined use of TMS and three complementary neuroimaging techniques, namely Electroencephalography (EEG), Positron Emission Tomography (PET) and functional Magnetic Resonance Imaging (fMRI). Although a number of excellent reviews on different methodological (e.g., Ilmoniemi and Kicić, 2010; Wagner et al., 2007) or more contentdriven aspects (e.g., Driver et al., 2009) of multimodal imaging involving TMS have already been published (for more specific references, see below), no extensive review of the types of paradigms used or research questions addressed exists to date. More specifically, we will focus on the observed local and remote neural effects of TMS as measured with EEG, PET, and fMRI to gain insights into the neurophysiological impact of TMS at the network level as reflected across these different neuroimaging modalities. By covering all these complementary forms of multimodal TMS research, one can systematically look into the question to what extent the acquired findings are in general agreement with each other. One major hurdle in doing so, however, is the fact that the already mentioned virtually infinite TMS parameter space has only been explored in a somewhat arbitrary fashion, and many studies therefore differ in a variety of ways which often makes direct comparisons difficult. Nevertheless, the amount of multimodal TMS studies has by now reached a critical mass that makes it worthwhile to see whether some general conclusions regarding local and remote neural effects of TMS can be drawn from the existing literature. To this end, we have systematically categorized and assessed all multimodal TMS publications retrievable via the PubMed database (http://www.ncbi.nlm.nih.gov/pubmed/), and references therein, based on the neuroimaging method used and the chosen TMS target site. For current purposes, only studies 


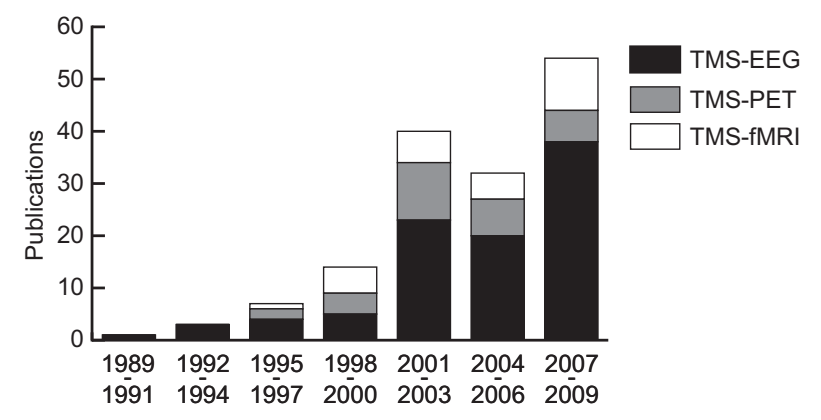

Fig. 1. Number of multimodal imaging publications involving TMS. The bar graph provides an overview of the number of publications on multimodal TMS research over the last 20 years (from 1989 to 2009; binned in clusters of 3 years; reviews not included), split up with respect to the three neuroimaging modalities covered in this review. Overall, 94 TMS-EEG, 30 TMS-PET, and 27 TMS-fMRI studies were retrieved, respectively representing $62.25 \%, 19.87 \%$, and $17.88 \%$ of the total number of 151 publications.

involving healthy volunteers were considered and only studies published before January 2010 were included. Note that even though not all considered studies are described in the main text, a complete library of the relevant literature that we could retrieve is provided as supplementary material (classified according to the combination of methods used). As shown in Fig. 1, the number of published papers using TMS in combination with one of the three main neuroimaging methods covered here has consistently increased over the years, amounting to an estimated total of around 151 studies at the time of writing. The main research lines and resulting findings described in these publications are summarized in the following sections, clustered according to the methods used and the chosen TMS target sites. This review thereby provides a structured overview of the status quo in this exciting field of research, in which significant progress has been made since the first multimodal TMS measurements were undertaken about two decades ago.

\section{Neural mechanisms of TMS}

As already mentioned, TMS relies on the principles of electromagnetic induction to generate electrical currents in neural tissue. The direct impact of a TMS pulse is limited to a patch of cortex of a few square centimeters. In addition, the induced field falls off exponentially with distance, meaning that superficially located tissue is excited more easily. The effective penetration depth of TMS is estimated to be $\sim 2 \mathrm{~cm}$ (e.g., Rudiak and Marg, 1994). To understand the resultant neural effects of TMS, it is important to determine the characteristics of the generated electromagnetic fields (Wagner et al., 2009), and current modeling efforts are focusing on the use of more realistic head models (e.g., taking into account local brain anatomy and the presence of different tissue types) in order to better describe the induced current distributions. In parallel, both modeling and in vitro work has examined the most likely loci of physiological excitation at the neuronal level, suggesting that particular cell compartments (e.g., fiber bends or the axon-cell body boundary) are preferentially affected and cell morphology therefore is a determining factor in whether a neural response is elicited (Radman et al., 2009). Whenever the right biophysical preconditions are met, the induced currents depolarize the cells' membrane, thereby opening voltagesensitive ion channels and actively initializing action potentials (for a discussion on models of transcranial neural stimulation, see Wagner et al., 2007). Even if no actual spiking activity is induced, TMS is thought to influence neural processing by more subtle modifications of other neuronal properties (e.g., excitation thresholds, alterations in spontaneous activity, synaptic efficacy, etc.).
However, the exact mechanisms involved remain elusive, foremost because TMS indiscriminately affects a rather large patch of cortex consisting of different cell types. Besides their divergent functional roles, distinct cell types may for example additionally have different response time constants or threshold characteristics (Pell et al., 2011), and such properties potentially vary across different cortical regions. Consequently, although all kinds of interactions between facilitatory and inhibitory processes may occur at different neural levels, only their overall net effect is commonly observable. The scarcely available data from animal models indicate that a single TMS pulse can set in motion a complex cascade of events: from initial facilitation of both spontaneous and visually evoked single-unit spiking activity (up to $500 \mathrm{~ms}$ ) to subsequent suppression (up to several seconds), with higher stimulation intensities leading to interruptive early suppression up to $200 \mathrm{~ms}$ post-TMS followed by rebound excitation before showing the late inhibition (Moliadze et al., 2003). This time course of initial facilitation followed by prolonged suppression after single pulse TMS has been suggested to also underlie the frequency-dependent effects of repetitive TMS protocols. More specifically, repetitive stimulation at higher frequencies could lead to synaptic potentiation when the presynaptic activity induced by the incoming TMS pulse coincides with the post-synaptic depolarization resulting from the preceding pulse. In contrast, low-frequency stimulation is hypothesized to result in synaptic depression when the incoming pulse arrives during the late inhibitory phase produced by the previous pulse (Funke and Benali, 2010). However, data from a similar study on the effects of short (1-4s) trains of repeated stimulation (at $1-8 \mathrm{~Hz}$ ) on spontaneous and evoked spiking activity (Allen et al., 2007) as well as some of the human neuroimaging studies discussed in the following sections suggest that such a strict dichotomy between high and low frequency rTMS is likely to be an oversimplification.

The most widely accepted view regarding repetitive TMS effects which outlast the period of stimulation is that the involved neural mechanisms are akin to phenomena reflecting synaptic plasticity, mainly long term potentiation/depression (LTP/LTD; Bliss and Lømo, 1973) of excitatory synaptic transmission (Thickbroom, 2007). Two recent reviews have critically assessed the link between rTMS and LTP/LTD (Hoogendam et al., 2010; Pell et al., 2011). Interestingly, several parallels could be identified. Beside the fact that both LTP/LTD induction and rTMS lead to prolonged changes beyond the stimulation period, other similarities include: sensitivity to the temporal pattern of the stimulation protocol, the dependence of the induced excitability changes on the preceding activation history (metaplasticity; Abraham and Bear, 1996), the shared influence on learning experiences, and findings from pharmacological and animal studies showing that rTMS affects neural processes related to the initiation and maintenance of synaptic plasticity (such as gene and protein expression, NMDA receptor functioning, etc.; see also Cheeran et al., 2010). Nevertheless, all of these findings only constitute indirect evidence linking rTMS to synaptic plasticity mechanisms, and certain divergences are also clear (Hoogendam et al., 2010; Pell et al., 2011). For example, the application of rTMS is much less potent in terms of the duration of after-effects or the consequences of cumulative stimulation than the protocols used to induce LTP/LTD, and of course both are applied at extremely different spatial scales (at the level of single neurons for LTP/LTD to whole cortical areas in rTMS). Recent complementary propositions hold that next to LTP/ LTD-like excitability changes in excitatory synaptic transmission (e.g., via summation of effects depending on the amount and rate of postsynaptic calcium influx, see Huang et al., 2011), modulations of inhibitory interneuron activity (Funke and Benali, 2010) and membrane potentials (Pell et al., 2011) are co-occurring reflections of the impact of rTMS. 

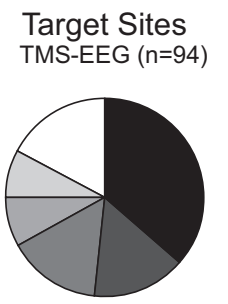

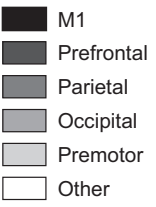

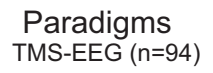

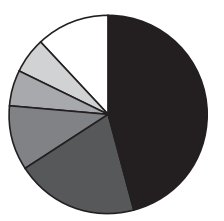

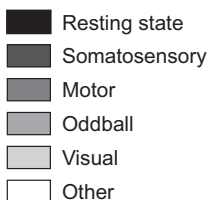

TMS Protocols TMS-EEG $(n=94)$

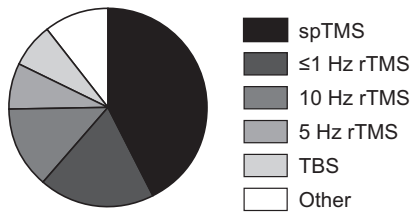

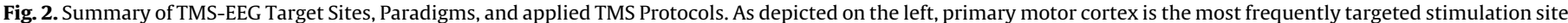

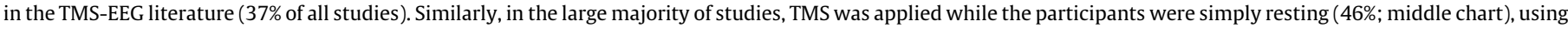

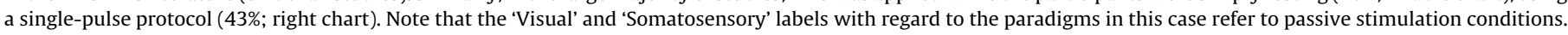
Grey-scale legends are rank-ordered by magnitude of the corresponding proportion, depicted in clockwise order in the respective pie charts.

Even though the neural mechanisms underlying TMS-induced effects are not yet fully understood and are likely to encompass mutually interacting phenomena, they lead to significant activation changes at the macro "neural systems" level that can be detected with current human neuroimaging methods, as described in detail below.

\section{Multimodal TMS research}

\subsection{TMS-EEG studies}

Its high temporal resolution and the fact that the recorded signal has its origin directly in electrical neural activity (contrary to PET and fMRI which rely on indirect haemodynamic signals) make EEG a suitable candidate for use in combination with TMS (see also Komssi and Kähkönen, 2006; Miniussi and Thut, 2009; Taylor et al., 2008; Thut and Miniussi, 2009). Initial attempts to characterize in vivo connectivity using TMS together with a limited number of EEG electrodes explored transcallosal (Cracco et al., 1989) and cerebello-frontal (Amassian et al., 1992) connections, revealing remote (i.e., relative to the TMS target site) responses with onset latencies around $10 \mathrm{~ms}$ post-TMS. Since these pioneering experiments, more than 90 TMS-EEG studies have been reported in the literature (see Fig. 2 for an overview of the relative proportions regarding chosen TMS target sites and experimental context across studies), rendering it the most commonly used technique in combination with TMS. The most prominent findings based on TMS-EEG research to date are discussed in more detail below.

\subsubsection{Target site: primary motor cortex (M1)}

TMS to the primary motor cortex (M1) can elicit brief muscle contractions in the hand contralateral to the targeted hemisphere (observable via so-called Motor Evoked Potentials (MEPs) recorded at electrodes located near the relevant muscle). The lowest TMS intensity required to produce such a contraction is referred to as the 'resting Motor Threshold' (rMT) or 'active Motor Threshold' (aMT) depending on whether the participant's hand muscle was relaxed or already contracted at the time point of determination. These individually determined thresholds are often used as a reference to report the applied intensity in the TMS literature, and will regularly be used in the same fashion here.

By applying single-pulse TMS (spTMS) slightly below rMT to primary motor cortex (M1) or visual cortex, and tracking the temporal evolution of the average evoked EEG potential maps, Ilmoniemi et al. (1997) could show that (1) the stimulated area produced an immediate response (reflecting the local "cortical reactivity" to TMS) and (2) that this activation rapidly spread to both adjacent ipsilateral sites as well as homologous contralateral areas within $20 \mathrm{~ms}$ (representing the revealed "connectivity" between the stimulated and the consequently activated brain areas). Using similar settings, Komssi et al. (2002) confirmed these latency ranges for the TMS-induced ipsi- and more diffuse contralateral spread in activation following left sensorimotor cortex stimulation. In addition, placing the coil $1 \mathrm{~cm}$ away from the optimal M1 target site changed the observed activation pattern, with more medial stimulation resulting in smaller contralateral responses.

Another way to quantify the impact of a TMS pulse is to calculate the Global Mean Field Amplitude (GMFA). This is a summary measure of the overall brain response as it highlights the deviations from the mean response across all recorded electrodes. At least four GMFA peaks have been consistently observed for both left and right M1 stimulation (at 15, 44, 102 and 185 ms post-TMS; Komssi et al., 2004). Changing the intensity of the TMS pulses only affected the amplitude of the peak responses, not the corresponding latencies or associated scalp distributions. Such intensitydependent modulations have been replicated for a wide range of stimulation intensities, and clear responses even appeared at an intensity of $40 \%$ of the MT for TMS over M1, underlining the sensitivity of EEG measures (Komssi et al., 2007). When focusing on single or predefined subsets of electrodes instead of the more general GMFA, characteristic waveforms - so-called TMS-Evoked Potentials (TEPs) - can be observed with similar morphologies across subjects (Komssi et al., 2004) and good separability and reproducibility across different sessions for three different TMS intensities (Lioumis et al., 2009). Several studies have provided converging results on these TEPs and the corresponding latency ranges. Initially, Paus et al. (2001a) observed P30, N45, and N100 peaks after M1 stimulation. ${ }^{3}$ Besides confirming the P30/N45/ N100 morphology, others have subsequently identified additional deflections such as the N15, P55, and P180 (Komssi et al., 2002, 2004), or further subdivisions (e.g., a N10/P14/N18 complex; Bonato et al., 2006). Interestingly, EEG responses to TMS can display state-dependent effects in the sense that the observed TEPs can vary depending on the current mind set of the participant: Nikulin et al. (2003) observed that the N100 TEP is weaker and peaks later when spTMS is applied to sensorimotor cortex during the preparation-phase of a visually cued movement compared to when TMS is administered in isolation (whereas the resulting MEP increased). Moreover, even simply showing the visual stimulus without requiring an associated motor response already modulated these N100 properties. A similar, though less pronounced, attenuation of the N100 is also present when stimulating the ipsilateral M1 relative to the finger to be moved (Kicić et al., 2008).

TMS can also influence or interact with the processing of incoming somatosensory information. In a series of studies, TMS over the contralateral sensorimotor cortex was combined with median nerve stimulation at the wrist to investigate modulations

\footnotetext{
${ }^{3}$ Note that the ' $\mathrm{N}$ ' and ' $\mathrm{P}$ ' prefixes, respectively, denote negative and positive deviations from baseline, and the added numbers represent the time in ms relative to the onset of TMS. Commonly, the latencies used to name the peaks are based on the recordings from the $\mathrm{Cz}$ electrode, and therefore might deviate for other electrode sites.
} 
of evoked potentials measured via EEG. The acquired data indicated that applying M1-spTMS up to $50 \mathrm{~ms}$ before the peripheral stimulation resulted in enhanced early EEG responses (Kujirai et al., 1993; Seyal et al., 1993). Enhanced responses to sensory input can also be found following the pairing of $0.1 \mathrm{~Hz}$ rTMS with peripheral stimulation (Tsuji and Rothwell, 2002). Moreover, whereas $1 \mathrm{~Hz}$ rTMS produces long-lasting suppression of subsequent sensory evoked EEG responses (Enomoto et al., 2001), continuous TBS enhanced these responses (while suppressing bilateral MEPs; Ishikawa et al., 2007) and $0.2 \mathrm{~Hz}$ rTMS did not induce any change (Urushihara et al., 2006). Trains of paired stimulation with a temporal asynchrony of $25 \mathrm{~ms}$ between peripheral and spTMS cortical stimulation can not only potentiate subsequently evoked MEPs, but also affect ensuing sleep patterns thought to reflect the induced cortical plasticity (De Gennaro et al., 2008). Alternatively, it has been shown that isolated trials of peripheral digit stimulation can lead to brief periods (tens of $\mathrm{ms}$ ) in which TMS-induced MEPs are suppressed. This attenuation is correlated with a reduction in N100 amplitude following spTMS, consistent with a cortical origin of this effect (Bikmullina et al., 2009). A related short-lasting type of state-dependence was demonstrated by changes in cortical reactivity due to the pairing of two TMS pulses to M1, where the first pulse leads to a suppressed response to the second pulse (Daskalakis et al., 2008; Fitzgerald et al., 2009).

Beside such analyses in the time-domain, one can also investigate the neural consequences of TMS by looking at the frequency content of the recorded EEG responses. For example, spTMS over M1 was shown to immediately induce $15-30 \mathrm{~Hz}$ ("beta" range) oscillations in the vicinity of the stimulation site, lasting for several hundred ms (Paus et al., 2001a). Additional research subsequently showed this effect was rather spatially specific (premotor TMS was less effective) and demonstrated that the observed fluctuations were strongly phase-locked to the onset of spTMS, suggesting a reset of ongoing oscillations (van der Werf and Paus, 2006). Others have reported similar short lasting $(<500 \mathrm{~ms})$ changes in oscillatory activity within both the alpha $(8-12 \mathrm{~Hz})$ and beta frequency band following spTMS to M1 (Fuggetta et al., 2005). These modulations only consistently occurred with suprathreshold TMS (mainly at 130\% rMT), were more pronounced ipsilateral to the stimulation site, and contrasted with alpha/beta frequency power decreases accompanying voluntary finger movements.

Next to the neural consequences of single pulse TMS applications as mainly discussed so far, several groups have also investigated the impact of prolonged repetitive TMS protocols as measured with EEG. During the continued application of $1 \mathrm{~Hz}$ rTMS (Brignani et al., 2008) and following short trains of $5 \mathrm{~Hz}$ rTMS (though not always significantly different from sham control stimulation; Fuggetta et al., 2008) to M1, enhancements in alpha and beta band power have been reported that were more pronounced ipsilateral to the stimulation site, with the former progressively increasing across successive stimulation blocks for electrodes C3 and P3 (Brignani et al., 2008). Other studies contrasting pre- vs. post-rTMS EEG epochs have mainly focused on post-rTMS changes in somatosensory evoked responses (as discussed above), spTMS responses (Esser et al., 2006; Huber et al., 2007; van der Werf and Paus, 2006), EEG components reflecting preparatory movement-related activity (Holler et al., 2006; Ortu et al., 2009; Rossi et al., 2000) and changes in coherence ${ }^{4}$ across recording electrodes (Oliviero et al., 2003; Plewnia et al., 2008;

\footnotetext{
${ }^{4}$ The more synchronous the activity between electrodes in a given frequency band (thought to reflect the interaction between distal sites through the "communication channel" a particular frequency band provides), the higher the resulting coherence.
}

Sağlam et al., 2008; Strens et al., 2002). Interestingly, the rTMSinduced changes in responses to spTMS and ongoing neural rhythms were dependent on the chosen rTMS frequency, although strict comparisons might be hampered by the fact that the total number of TMS pulses was not matched across studies. While $0.6 \mathrm{~Hz}$ rTMS led to suppressed cortical responses to spTMS (van der Werf and Paus, 2006), $5 \mathrm{~Hz}$ rTMS resulted in potentiated responses to spTMS in nearby premotor cortex (Esser et al., 2006), subsequently also reflected in elevated slow wave activity during the first $30 \mathrm{~min}$ of sleep (Huber et al., 2007). With respect to ongoing neural oscillations, Strens et al. (2002) found ipsilateral alpha band coherence increases between primary and premotor cortex that lasted up to $25 \mathrm{~min}$ post- $1 \mathrm{~Hz}$ rTMS both during rest and active muscle contraction, and shorter lasting interhemispheric increases during muscle contraction episodes. Conversely, Oliviero et al. (2003) report largely ipsilateral decreases in alpha band power immediately following $5 \mathrm{~Hz}$ rTMS to left M1 (only short lasting and no longer visible 25 or 50 min later), restricted to periods of active muscle contraction. In other words, not only the main direction of the effect (i.e., increases vs. decreases in coherence), but also the time window (i.e., still noticeable vs. absent 25 min post-rTMS) and relation to the activation state of the muscular system (i.e., occurring exclusively during active muscle contraction or also during rest) varied considerably based on the chosen rTMS frequency.

Results such as these speak to the versatility of (r)TMS as a neuroscientific tool, but also should make users aware of the difficulties in foreseeing the neural effects of any given combination of TMS parameters (see below).

\subsubsection{Target site: frontal cortex}

In one of the few studies contrasting different TMS target sites in a within-subject design, Kähkönen et al. (2004) recorded EEG responses to single TMS pulses targeting either left M1 or prefrontal cortex (PFC) during rest. While keeping TMS intensity fixed (100\% rMT), PFC TMS led to smaller GMFA responses compared to M1 (particularly up to $130 \mathrm{~ms}$ post-TMS). Even though their reactivity differed in amplitude, the measured EEG responses evoked by TMS to both target sites were significantly correlated. Making use of MRI-navigated TMS, the same group later identified five consistently occurring EEG peaks (at 27, 39, 52, 105 and $193 \mathrm{~ms}$ at Fz/FCz) after PFC spTMS (Kähkönen et al., 2005). In addition, they observed an almost linear relation between PFC TMS intensity and the overall evoked brain response, without any changes in the peak latencies or the associated scalp topography. Four studies to date investigated changes in the frequency domain following PFC rTMS during rest, respectively reporting increases in theta $(4-7 \mathrm{~Hz}$, contralaterally; Schutter et al., 2001), alpha (Grossheinrich et al., 2009; Okamura et al., 2001) and delta (13.5 Hz; Griskova et al., 2007) frequency power post-TMS. However, these results are difficult to compare as several rTMS parameters differed substantially, as did the analyzed time window post-TMS. Increases in prefrontal theta frequency power (Schutter and van Honk, 2006) and shifts in prefrontal hemispheric asymmetries in the $30-50 \mathrm{~Hz}$ range (Schutter et al., 2003) have also been reported following, respectively, spTMS and $25 \mathrm{~Hz}$ rTMS to the cerebellar midline. Conversely, applying a single conditioning pulse to prefrontal cortex significantly attenuated the local EEG response to a test TMS pulse administered $100 \mathrm{~ms}$ later in all tested frequency bands, including the gamma range $(30-50 \mathrm{~Hz}$; Farzan et al., 2009). The only other frontal region that has been targeted to study prolonged rTMS effects during rest is the right Frontal Eye Field (FEF). Here, continuous TBS resulted in increased synchronization across 6 predefined electrode positions in several frequency bands, with changes being more pronounced in the stimulated right hemisphere up to 1 hour post-TBS (Schindler et al., 2008). 
There are also several studies on task-related neural activity changes due to frontal (r)TMS interventions. Modulations of neural activity following TMS of the PFC have mainly been charted in the context of the so-called 'oddball' paradigm, in which an auditory or visual deviant (the oddball) is presented amidst numerous repetitions of an identical stimulus (the standard). Following short trains of left frontal $10 \mathrm{~Hz}$ rTMS, a decrease in the P200 latency, but an increase in the P300 latency has been found in the response to an auditory oddball (Jing et al., 2001a,b), accompanied by an amplitude attenuation of the N100, P200 and P300 EEG components (Jing et al., 2001b). A similar increase in the auditory P300 latency was observed after $15 \mathrm{~min}$ of left PFC $1 \mathrm{~Hz}$ rTMS (Hansenne et al., 2004). In contrast, using a visual oddball paradigm, Evers et al. (2001) report a decrease in the P300 latency after 3 short trains of $20 \mathrm{~Hz}$ rTMS over left, but not right PFC (in combination with a decrease in reaction time to the target stimulus). The only other task-related PFC-rTMS study investigated the effect of $20 \mathrm{~Hz}$ rTMS in the context of an N-back working memory paradigm, and revealed increased gamma oscillatory activity post-rTMS, yet no effects on behavioral performance (Barr et al., 2009).

Targeting premotor cortex, fifteen minutes of subthreshold $0.9 \mathrm{~Hz}$ rTMS led to a reduction of the normally observed taskrelated decreases in alpha/beta power in a simple motor task, accompanied by increases in the coherence among cortical motor areas in the upper alpha band and decreases in the coherence between cortical and peripheral muscle responses that lasted for 15 min post-rTMS (Chen et al., 2003).

Other frontal regions that have been targeted during active task performance are the dorsal Medial Frontal Cortex (MFC) and FEF. In a classical Eriksen flanker task (where target stimuli are flanked by other stimuli that prime a response that is either compatible or incompatible with the required response to the target stimulus), $1 \mathrm{~min}$ of $0.9 \mathrm{~Hz}$ rTMS to MFC produced a decrease in behavioral error-correction rate, an attenuation of the Error-related Negativity and an enhanced later positivity in trials in which subjects made an error (Rollnik et al., 2004). Conversely, short trains of $10 \mathrm{~Hz}$ TMS over left MFC led to increased error rates on incongruent trials in which a right hand response should have been suppressed. Incongruent trials in which the correct response was given (i.e., the right hand response was successfully inhibited), nevertheless were associated with TMS-induced enhancements in the ERP difference waves between congruent and incongruent trials at 180-280 ms after stimulus onset, reflecting increased response conflict (Taylor et al., 2007a). Again applying short $10 \mathrm{~Hz}$ TMS trains, but now to right FEF, Taylor et al. (2007b) could show remote TMS effects in ipsilateral occipito-parietal cortex in a covert attention orienting task. More specifically, FEF-TMS administered between the presentation of a cue and the to-be-attended stimulus led to greater negativity at this posterior site up to $200 \mathrm{~ms}$ post stimulus onset. TMS therefore affected both the ongoing visual cortical activity prior to visual stimulus onset, as well as the evoked visual response itself. Short rTMS trains to right FEF can also disrupt anticipatory alpha rhythm desynchronization, which in normal circumstances can be detected in visual cortex when subjects are cued towards a future target location. By applying $20 \mathrm{~Hz}$ rTMS for $125 \mathrm{~ms}$ to either FEF or the intraparietal cortex following a spatial cue, Capotosto et al. (2009) interfered with this preparatory alpha desynchronization, thereby slowing down the identification of visual target stimuli presented $2 \mathrm{~s}$ later. Another elegant demonstration of the functional relevance of the connectivity between the FEF and remote visual cortex was provided by Morishima et al. (2009). In this study, subjects had to perform a motion direction or face-gender discrimination task on superimposed images of faces and moving gratings. As the stimuli were identical for both tasks, differences in processing could only be related to top-down modulations based on the given task instructions. Interestingly, the posterior visual area to which a single subthreshold FEF-TMS pulse was most efficiently transmitted, depended on the stimulus feature subjects were currently attending. The strength of this area-specific spTMS transmission furthermore depended on the level of attentional preparation and was related to behavioral response latencies (i.e., higher downstream activations following spTMS were found when comparing fast versus slow behavioral responses), reflecting the benefits of the established top-down biasing signal originating in the FEF. Instead of influencing the neural processing in the targeted brain area, TMS was used here to probe the current task-induced state of connectivity between distantly located nodes within a cortical network.

Complimentary to this task-dependent modulation of the current brain state, one can also exploit natural fluctuations in such states and again use TMS to probe and describe the underlying network's properties. More specifically, Massimini et al. (2005) assessed the impact of spTMS to premotor cortex while subjects were either awake or asleep. Intriguingly, EEG responses to spTMS during sleep were stronger in amplitude (although high-frequency components were dampened), more short-lasting, locally confined, and more stereotyped compared to responses evoked during wakefulness (Massimini et al., 2005). In other words, sleep led to a "breakdown" of transcallosal and longrange cortical connectivity as the initial response did not propagate to more distant brain areas (although undifferentiated global slow waves can be elicited during sleep, e.g., when stimulating sensorimotor cortex (Massimini et al., 2007)). Finally, the superior frontal gyrus was one of the exemplar regions in which spTMS could be shown to consistently evoke fast beta/gamma-band (21$50 \mathrm{~Hz}$ ) oscillations while subjects were at rest (Rosanova et al., 2009). The frequency band modulation revealed using this perturb-and-record approach systematically varied depending on the TMS target site: next to the fast beta/gamma-band responses in frontal cortex, occipital and parietal spTMS caused changes in the alpha and beta band, respectively. These variations were suggested to reflect each area's 'preferred' or 'natural' frequency, expressed both locally and as the dominant frequency in the overall brain response to TMS across a wide range of stimulation intensities. Moreover, locally observed frequency changes were biased towards a region's preferred frequency even if that region was only indirectly stimulated (through connections with another distal site that was currently targeted). The dominant frequency evoked at the target site therefore interacts with the evoked frequency modulations at distal sites (Rosanova et al., 2009).

\subsubsection{Target site: visual cortex}

While varying the time point of spTMS delivery with respect to the onset of a visual stimulus, Thut et al. (2003a) investigated the effects of TMS to the occipital pole at $120 \%$ of the individual phosphene threshold (i.e., the intensity at which spTMS can elicit a brief percept of a flashing light) on Visual Evoked Potentials (VEPs) in the EEG. Results indicated that spTMS at visual stimulus onset led to VEPs that were indistinguishable from trials in which no TMS was applied at all, given that spTMS artifacts were removed via subtraction of the signal obtained in TMS-only trials (i.e., in which no visual stimulus was presented). In contrast, administering a pulse during the build-up or peak of the VEP's P1 component changed the evoked response's waveform (reduced area under the curve without reduction in amplitude). Moreover, the same spTMS also affected the subsequent negative VEP-component, suggesting that the effect of the short (i.e., sub-millisecond) TMS pulse lasted up to $100 \mathrm{~ms}$. Other studies employing $\geq 10 \mathrm{~min}$ of $1 \mathrm{~Hz}$ rTMS have reported somewhat contradictory findings. On the one hand, 
occipital rTMS did not cause any modulation in the previously mentioned early VEP components in a study by Thut et al. (2003b). Instead, rTMS prominently reduced the visual-induced alpha desynchronization and moderately enhanced a later VEP component (P200) to right visual field stimulation whenever a right hand response was required (Thut et al., 2003b). These effects were restricted to the targeted left hemisphere, were no longer detected 20 min post-rTMS and probably reflect interference with corticocortical gating during visuomotor integration (i.e., higher-order functions beyond mere stimulus processing per se). On the other hand, rTMS-related decreases in early VEP component amplitudes have been reported up to $20 \mathrm{~min}$ following $15 \mathrm{~min}$ of $1 \mathrm{~Hz}$ rTMS (Fumal et al., 2003), together with a weakening of the habituation that normally occurs following repeated visual stimulation (an effect that might be extended in duration by applying the same rTMS protocol across five consecutive days; Fumal et al., 2006). Both these effects were much weaker or completely absent following $10 \mathrm{~Hz}$ rTMS with the same total number of pulses in the same subject group (Fumal et al., 2003), Another study also identified an attenuation of the earliest VEP component following $20 \mathrm{~min}$ of $1 \mathrm{~Hz}$ rTMS in the context of the Eriksen Flanker task (Schutter and van Honk, 2003). However, only the contrast with sham, but not vertex rTMS was significant here and the time window of the induced changes was not investigated. Again, the origin of the discrepancies between studies reporting the presence vs. absence of VEP amplitude modulations following $1 \mathrm{~Hz}$ rTMS remains unknown, although a likely possibility is that the observable rTMS effect is partially dependent on the type of visual stimulus used and the total number of TMS pulses applied. The findings discussed here do however converge regarding the absence of any TMS effect on the observed latencies of the investigated ERP components, irrespective of the details of the used TMS protocol.

\subsubsection{Target site: parietal cortex}

Similar to the M1 and prefrontal target sites discussed above, spTMS at rest directed at the parietal cortex elicits a distinct TEP waveform consisting of four deflections between 43 and $158 \mathrm{~ms}$ post-TMS (Zanon et al., 2010). Moreover, spTMS induced activation increases in contralateral frontal and ipsilateral temporo-occipital cortex, starting 100 and 170 ms following stimulation, respectively. The effects of parietal TMS on task-related activity have only been investigated in three studies so far. During a visual search task, spTMS $100 \mathrm{~ms}$ after search array onset delayed reaction times in 'target present' trials, and eliminated the early phase of the attention-related N2pc ERP component (Fuggetta et al., 2006). By applying $10 \mathrm{~Hz}$ parietal-rTMS for $3 \mathrm{~s}$ in the delay phase of a spatial working memory recognition task, response accuracy was increased while reaction times to memory probes were unaffected (Hamidi et al., 2009). Interestingly, whereas rTMS on average did not change delay-related alpha-band power increases, considerable changes in opposite direction (i.e., TMS-related alpha power increases vs. decreases) were found for individual subjects. Moreover, these individual differences in alpha-power modulation were predictive of the concomitant effect of rTMS on behavioral measures: the stronger the rTMS-induced increase in alpha-band power, the more errors were committed (Hamidi et al., 2009). The cortical sources of this effect were estimated to lie in a distributed network including posterior frontal, ventral parietal and lateral occipital cortices and the hippocampus. A noteworthy related finding was reported by Klimesch et al. (2003), when applying a short rTMS train of 24 pulses at the individually-defined upper alpha frequency to the right intraparietal sulcus before subjects engaged in a mental rotation task. While enhancing alpha power in the resting EEG, the TMS intervention subsequently led to increased alpha-desynchronization during the mental rotation task trials, together with improvements in performance. These effects were not observed when applying 24 rTMS pulses at a higher or lower frequency, underlining the specificity of the induced effects.

Analogous to some of the studies mentioned in the section on M1-TMS, trains of spTMS over primary somatosensory cortex preceded by peripheral median nerve stimulation led to enhanced early somatosensory EEG responses (Wolters et al., 2005; see Litvak et al., 2007 for related source analysis and behavioral changes), as did single pairings of cortical and peripheral stimulation presented either simultaneously (Schürmann et al., 2001) or with offsets up to $40 \mathrm{~ms}$ (Raij et al., 2008; with strongest modulations following TMS to secondary somatosensory cortex). Whereas $0.9 \mathrm{~Hz}$ rTMS to somatosensory cortex left evoked responses to median nerve stimulation unaltered (Satow et al., 2003), the inhibition that is normally visible for the second of two peripheral stimulation events was substantially suppressed following $5 \mathrm{~Hz}$ rTMS (Ragert et al., 2004). After applying rTMS at 1 or $10 \mathrm{~Hz}$, the initial burst of the highfrequency cortical response to median nerve stimulation was increased $(1 \mathrm{~Hz})$ or unaltered $(10 \mathrm{~Hz})$, while the later burst was decreased vs. increased, respectively (Restuccia et al., 2007). Finally, different TBS protocols that are assumed to be facilitatory (intermittent TBS) or suppressive (continuous TBS) resulted in the expected enhanced (Katayama and Rothwell, 2007) and attenuated (Ishikawa et al., 2007) cortical somatosensory-evoked responses following median nerve stimulation. Contrastingly, however, all types of TBS led to undifferentiated attenuated responses to cutaneous laser stimulation to the subjects' hands, without differences in concomitant subjective pain perception compared to sham stimulation (Poreisz et al., 2008).

\subsubsection{Summary TMS-EEG}

The presented overview shows that TMS can elicit clear physiological responses in the stimulated cortex, reflected both by evoked potential waveforms in the time-domain as well as modulations in the frequency content of the measured EEG. The observed response to TMS appears to be quite consistent across multiple repetitions and is oftentimes more pronounced in the ipsilateral hemisphere, but its exact properties are highly dependent on the chosen target site and the parameters of the applied TMS protocol. In general, applying TMS with higher intensity increases the strength of the neural response, while leaving its onset, morphology and scalp-distribution unchanged. EEG additionally proved to be a sensitive measure of TMS-induced changes in ongoing activity (e.g., revealing changes due to subthreshold spTMS or showing post-rTMS changes even though the corresponding MEPs appear unaltered), underlining its suitability to directly compare the neural consequences of different TMS protocols and settings. Furthermore, despite the method's low spatial resolution and the assumptions made during source localization attempts, EEG data support the notion of spreading activation from the stimulated target site to other more distant cortical network nodes. Interestingly, both the local and remote TMS-induced changes are task- and state-dependent, showing that TMS can really interact with ongoing cognitive processing or homeostatic states. Moreover, although results are not always consistent, EEG measurements following application of rTMS have provided evidence for prolonged (i.e., beyond the time window of stimulation itself) modulations of spontaneous as well as externally triggered cortical activity (see also Thut and PascualLeone, 2010). This nicely complements MEP-based quantifications of cortical excitability changes following rTMS, because EEG emphasizes the cortical origin of the observed effects and is suitable to chart the impact of rTMS across the entire cortex (compared to MEPs which are only observable following motor cortex stimulation). 
Target Sites
TMS-PET $(n=30)$

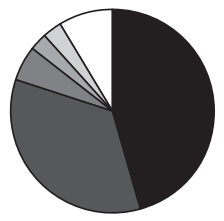

Paradigms

TMS-PET $(n=30)$

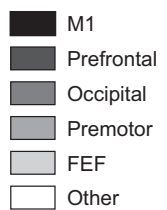

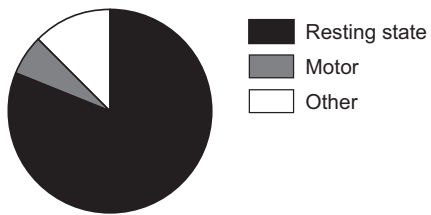

TMS Protocols

TMS-PET $(n=30)$

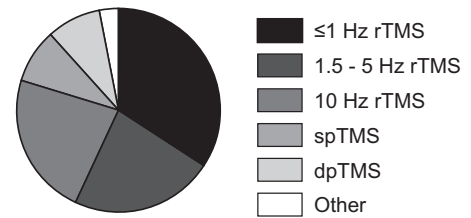

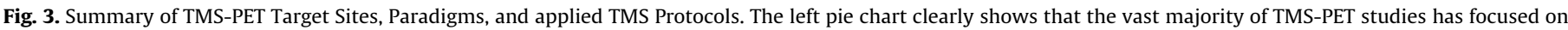

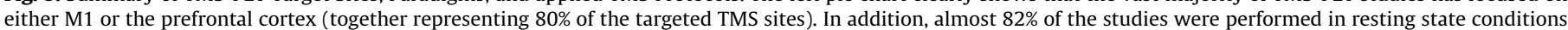
(middle chart), and rTMS protocols up to $10 \mathrm{~Hz}$ were most commonly used (right chart).

Finally, it should be noted that there is quite some variability in the methods used for TMS coil positioning across studies (possibly contributing to seemingly inconsistent results, especially for cortical regions that do not elicit readily observable peripheral responses), and that there are relatively few studies focussing on behaviorally relevant TMS-induced brain changes in the context of active task performance. To paraphrase Thut et al. (2003a, p. 2079): it remains to be determined under what conditions the alteration of functional neuronal activity translates into behavioral effects, as the neurophysiological changes in the magnetically stimulated cortex do not necessarily mirror consequences in cognitive processing.

\subsection{TMS-PET studies}

In contrast to EEG, PET imaging provides activation maps most commonly reflecting changes in cerebral blood flow (rCBF) or glucose metabolism (depending on the radioactive tracer used), covering both cortical and subcortical structures with higher spatial, yet limited temporal resolution (see also Paus, 2005; Siebner et al., 2009). The first study combining TMS with PET was published by Paus et al. (1997), investigating local and remote effects of TMS over left FEF. Since then, around 30 TMS-PET studies have been published, mainly focusing on brain networks at rest ( $\sim 80 \%$ of the reports; see also Fig. 3 ), as detailed below.

\subsubsection{Target site: primary motor cortex}

The primary motor cortex (M1) by far is the most frequently stimulated cortical site in the existing TMS-PET literature, targeted in almost $50 \%$ of the published papers.

Already in 1997, Fox et al. reported a continuous local rCBF increase under the coil during $30 \mathrm{~min}$ of $1 \mathrm{~Hz}$ rTMS, which persisted for at least $10 \mathrm{~min}$ after TMS offset. Even though the image acquisition parameters did not allow whole brain coverage, activity in remote sites was found to covary with the targeted left M1 activity. In stark contrast, Paus et al. (1998) only found decreases in $\mathrm{rCBF}$, both locally and at remote brain regions, when increasing the number of pulse trains of $500 \mathrm{~ms} 10 \mathrm{~Hz}$ rTMS applied over $60 \mathrm{~s}$. These initial studies already clearly illustrate the impact that changes in rTMS settings can have, and exemplify the wide variety of protocols used (Frequency: $1 \mathrm{~Hz}$ vs. $10 \mathrm{~Hz}$; Pulse mode: continuous vs. in trains, Total Number of Pulses: 1800 vs. 25-150 pulses; Intensity: 120\% rMT (suprathreshold) vs. 70\% maximum stimulator output (subthreshold)).

Most subsequent studies have reported TMS-induced increases in local activity beneath the coil (e.g., Siebner et al., 1998, 2001a; Ferrarelli et al., 2004), although it should be noted that $1-5 \mathrm{~Hz}$ rTMS protocols were employed in all of these studies. Such increases most likely do not reflect artifacts of feedback processes due to peripheral muscle contractions induced via suprathreshold TMS, as enhanced neural activity can similarly be found following subthreshold rTMS (Rounis et al., 2005; Siebner et al., 2000), and can outlast the actual time window of stimulation (e.g., Fox et al.,
1997; Siebner et al., 2000). Local brain activity was positively correlated with rTMS intensity (Speer et al., 2003a; Fox et al., 2006). Systematically varying the frequency of rTMS (between 1 and $5 \mathrm{~Hz}$ ) similarly revealed a positive correlation with local rCBF at rest (Siebner et al., 2001b), although this relationship might not hold in the context of active task performance (see Rounis et al., 2005).

But what about TMS-induced remote effects? As it turns out, effects at distal sites are reported in all of the reviewed studies. Besides the obvious auditory cortex activation whenever the TMS coil's click sound was audible, the vast majority of remote effects was observed in the motor network, both in connected cortical (e.g., SMA; Siebner et al., 2000), as well as subcortical (e.g., putamen; Strafella et al., 2003) nodes. ${ }^{5}$ In a direct comparison of responses to spTMS after $1 \mathrm{~Hz}$ rTMS to either M1 or premotor cortex, Chouinard et al. (2003) observed that although both treatments resulted in similarly suppressed MEPs, the network of distant regions affected by M1-rTMS was more confined (i.e., not including prefrontal or parietal activation changes). Interestingly, $1 \mathrm{~Hz}$ rTMS to M1 leads to increased activation of the dorsal premotor cortex in the non-stimulated hemisphere and changes in inter-areal connectivity during motor task execution post-rTMS, revealing possible coping mechanisms to fulfill current task demands (Lee et al., 2003). Although the generally revealed connectivity patterns to plausible functionally related and/or anatomically connected regions is reassuring, the nature of the impact TMS has on remote sites is quite diverse. First, the exact constellation of the affected network varies considerably, which is somewhat surprising given that the same network node (namely M1) was targeted in all studies. For example, co-activation of the contralateral premotor cortex has been reported by Siebner et al. (2001a), whereas other studies only found a trend here (Ferrarelli et al., 2004) or no change at all (Siebner et al., 2001b). Second, the direction of the TMS-induced changes for a given region is not consistent across studies. Even when the same region is repeatedly identified as a site showing remote effects of TMS over M1 in separate experiments, the actually elicited response is far from predictable. For example, activity modulations in the primary motor cortex contralateral to the stimulation site have been reported on several occasions. However, the response profiles go in opposite directions as both increases (Ferrarelli et al., 2004; Siebner et al., 2000) as well as decreases (Fox et al., 1997, 2006) in activity were observed. Third, even within a given experiment, the effect that TMS has on distal sites is not necessarily homogeneous throughout the affected network. Although some studies reported rather uniform responses (Paus et al., 1998), others found diverging effects of the same TMS protocol at different distal sites (Siebner et al., 2001b; Speer et al., 2003a).

\footnotetext{
${ }^{5}$ Although one should note that the analyses were oftentimes restricted to predefined 'regions-of-Interest' within this particular network of areas, somewhat limiting the method's full exploratory power.
} 


\subsubsection{Target site: frontal cortex}

To date, 12 TMS-PET studies have been published in which the prefrontal cortex was targeted. In the large majority of cases, the effects of TMS were studied while the subjects were at rest (i.e., did not engage in any particular task). When stimulating the left dorsolateral PFC, activity decreases in the vicinity of the TMS coil were observed after double-pulse (dpTMS; Paus et al., 2001b) and $1 \mathrm{~Hz}$ rTMS (Ferrarelli et al., 2004; Speer et al., 2003b). On the other hand, TMS over right PFC mainly seems to lead to local activity increases (for $1 \mathrm{~Hz}$ rTMS: Eisenegger et al., 2008; Ferrarelli et al., 2004; for $10 \mathrm{~Hz}$ rTMS: Knoch et al., 2006). Not all data support such a simple dichotomy though, as Knoch et al. (2006) additionally report local increases after both $1 \mathrm{~Hz}$ and $10 \mathrm{~Hz}$ rTMS over left PFC, and no local changes at all after right PFC rTMS at $10 \mathrm{~Hz}$. The exact reason for such discrepancies remains unclear.

Again, the most consistently reported finding across studies is that several other brain areas also change their response profiles following TMS to either left or right PFC. In particular, remote activity modulations are frequently observed in the anterior cingulate cortex (e.g., Ohnishi et al., 2004a; Speer et al., 2003b), anterior insula (e.g., Ferrarelli et al., 2004; Speer et al., 2003b), premotor cortex (e.g., Eisenegger et al., 2008; Knoch et al., 2006), the subcortical structures of the basal ganglia (e.g., Ferrarelli et al., 2004; Ohnishi et al., 2004a; Speer et al., 2003b), parahippocampal gyrus (e.g., Eisenegger et al., 2008; Knoch et al., 2006; Paus et al., 2001b) and cerebellum (e.g., Ferrarelli et al., 2004; Speer et al., 2003b). Besides the parahippocampal gyrus, these regions generally showed increased blood flow, although exceptions have been found for each case. Similar to the findings for M1-rTMS described above, increasing the PFC-rTMS intensity can lead to both parametric activation increases and decreases at remote sites (Speer et al., 2003b). However, in contrast to the intensitydependent increases following M1-rTMS (Speer et al., 2003a), the local response to PFC-rTMS showed a negative correlation with rTMS intensity level (Speer et al., 2003b).

Next to these effects during rest, activation modulations following PFC-rTMS have also been found during active task engagement. Local rCBF responses were suppressed following trains of $4 \mathrm{~Hz}$ rTMS to either left or right dorsal PFC during a verbal '2-back' working memory task, with right PFC-rTMS additionally lowering activation in bilateral parietal cortex despite similar behavioral deficits compared to left PFC-rTMS (Mottaghy et al., 2000). After $1 \mathrm{~Hz}$ rTMS to left dorsolateral PFC, decreases in glucose metabolism have been found in the left superior frontal gyrus (compared to sham rTMS), while occipital glucose metabolism increased during an auditory discrimination task (Kimbrell et al., 2002).

In addition to regional blood flow changes, PET can be used as a highly sensitive tool to monitor other, biochemical markers of brain function. For instance, binding of the specific $D_{2}$ dopamine receptor ligand $\left[{ }^{11} \mathrm{C}\right.$ ]raclopride can index the level of extracellular dopamine in regions where this neurotransmitter is abundantly found (i.e., mainly the basal ganglia), whereas the $\left[{ }^{11} \mathrm{C}\right]$ FLB 457 ligand provides sufficient signal-to-noise to measure dopamine levels throughout the cortex, even in brain regions with low $\mathrm{D}_{2}-$ dopamine receptor density. Increases in extracellular dopamine were observed in the left (but not right) basal ganglia (Strafella et al., 2001) and anterior cingulate and medial orbitofrontal cortex (Cho and Strafella, 2009) after left dorsolateral PFC $10 \mathrm{~Hz}$ rTMS. Likewise, changes in the synthesis of serotonin (by using $\left[{ }^{11} \mathrm{C}\right]-$ alpha-methyl-tryptophan trapping as a proxy) in remote limbic areas have been reported following left dorsolateral PFC (but not occipital) $10 \mathrm{~Hz}$ rTMS (Sibon et al., 2007). Finally, hemispheric asymmetries were revealed by applying TBS to either left or right dorsolateral PFC before letting subjects perform the Montreal Card Sorting Task (a neuropsychological task to test planning and set- shifting functions): only left-sided TBS led to impaired performance accompanied by reduced dopamine release in the ipsilateral putamen and contralateral caudate (Ko et al., 2008).

Two other frontal cortex sites have been targeted while combining TMS and PET, namely the MFC and the FEF. Stimulating the former led to increases in $\mathrm{rCBF}$ in mid-dorsal anterior cingulate cortex (accompanied by activation decreases in adjacent cingulate areas) and rCBF changes in a widespread network of distal (sub)cortical regions (Hayward et al., 2007). Regarding the FEF stimulation, Paus et al. (1997) found only positive correlations between the number of administered TMS pulses and the amplitude of both local and remote rCBF responses. This finding is interesting as M1 TMS in the same subjects and with identical TMS parameters actually resulted in only negative correlations between the number of TMS pulses and rCBF in a different set of areas (Paus et al., 1998), as discussed above. While underlining the specificity of evoked TMS effects depending on the chosen target site, these results also make clear that it is difficult to extrapolate earlier findings on the systems level impact of TMS to other target sites.

\subsubsection{Summary TMS-PET}

Overall, the existing TMS-PET literature conveys three important conclusions. First, without any exceptions, all published reports provide converging evidence on TMS-evoked activation changes in network nodes distal from the stimulated target site. Despite a wide range of applied stimulation protocols, varying time points of PET acquisition relative to TMS, and issues related to limited spatial coverage, rather small subject samples or suboptimal target site determination, remote effects were consistently revealed and have substantially contributed to a reconsideration of how TMS manipulations should be interpreted. Second, a coil displacement of only a few centimeters can drastically change the observed activation patterns (even in within-subject designs), clearly indicating that the impact of stimulation is highly locationdependent and therefore corroborating the specificity of TMS as a neuromodulator. Finally, both the local and remote impact of TMS over a given target site are highly dependent on the exact stimulation protocol used. However, our current understanding of the complex interactions between target site, stimulation parameters and functional state of the system is unfortunately still limited.

\subsection{TMS-fMRI studies}

Similar to EEG and PET, fMRI has its own benefits and drawbacks as a method to non-invasively measure human brain activity. Most importantly, it is superior with respect to the spatial detail that can be achieved in the resulting images (in the $\mathrm{mm}$ range). Compared to PET, spatial coverage of the whole brain can be achieved relatively fast (in the order of seconds), and repeated measurement sessions are feasible without imposing health risks (as no radioactive substances are involved). Conversely, fMRI only provides an indirect measure of neural activity (via haemodynamic responses) and can impose several practical limitations (due to space constraints, the strong magnetic field involved, susceptibility to image artifacts, etc.), also affecting the concurrent use of TMS (see e.g., Bestmann et al., 2008a). Nevertheless, the combination of TMS and fMRI has been very fruitful (Driver et al., 2009; Ruff et al., 2009a; see also Fig. 4), as discussed in the following sections.

\subsubsection{Target site: primary motor cortex}

The feasibility of combining (or to be more accurate: interleaving) TMS and fMRI was first demonstrated by Bohning et al. (1998), in an experiment in which spTMS (at 110\% rMT) led to significant activation increases in the targeted M1. The same 
Target Sites
TMS-fMRI $(n=27)$

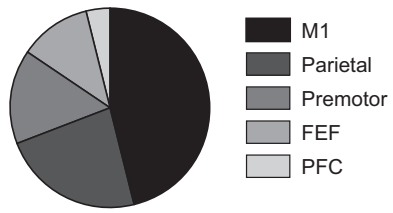

Paradigms

TMS-fMRI $(n=27)$

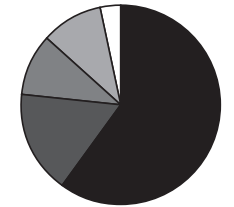

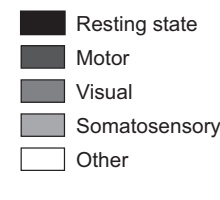

TMS Protocols TMS-fMRI $(n=27)$

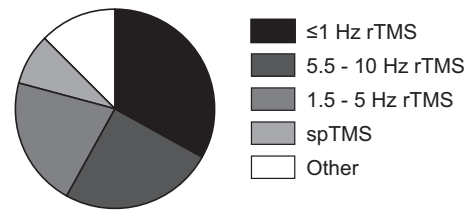

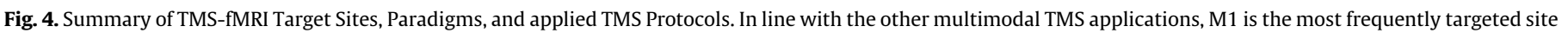

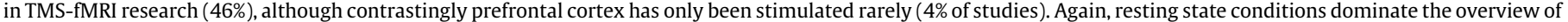

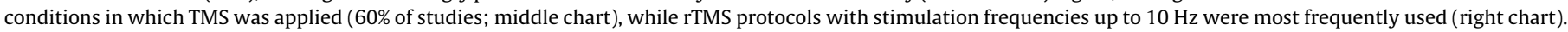
Note though that in TMS-fMRI research, such rTMS protocols are usually applied in an event-related fashion comprising short trains of stimulation.

authors subsequently illustrated intensity-dependent variations in activation strength (indexed by the number of activated voxels) by comparing $80 \%$ vs. $110 \%$ rMT TMS both near the targeted M1 (though not directly underneath the coil, possible due to a local decrease in signal-to-noise ratio) and in a small number of more remote regions such as the contralateral M1 and the ipsilateral cerebellum (Bohning et al., 1999). Furthermore, the elicited motor cortex activation showed a typical haemodynamic response profile (bilaterally; Bohning et al., 2000a) that was similar to the BloodOxygenation-Level-Dependent (BOLD) response measured during volitional movement (Bohning et al., 2000b), portraying a linear increase in amplitude and duration with increasing train length (Bohning et al., 2003). However, these findings were based on the application of TMS at suprathreshold intensities, by definition regularly leading to peripheral muscle contractions in the subjects' hand that constitute a confounding factor when trying to interpret the acquired imaging data (as local BOLD changes due to TMS could no longer be dissociated from cortical processing of the afferent sensory information due to the induced muscle contraction). In fact, even though axonal depolarization was probably reached at the TMS target site, neither subthreshold rTMS over M1, nor suprathreshold rTMS over adjacent lateral premotor cortex resulted in detectable BOLD responses in a number of considered motor structures (Baudewig et al., 2001, using $1 \mathrm{~s}$ of $10 \mathrm{~Hz}$ rTMS). This suggests that the previously observed activations after suprathreshold TMS to M1 mainly reflect the cortical processing of afferent input caused by the induced muscle contractions. Further support for this conclusion was provided by Bestmann et al. (2003), showing that $10 \mathrm{~s}$ of rTMS at intensities that did not result in peripheral muscle contractions led to enhanced BOLD responses in the supplementary and premotor cortices, but not in the directly stimulated M1/S1 cortex (see Hanakawa et al. (2009) for similar intensity-dependent remote activation changes based on spTMS). Interestingly, the contralateral right M1 showed activation decreases in all included conditions, an effect observed in some studies (e.g., Bestmann et al., 2004; Kemna and Gembris, 2003), but not all (e.g., Bohning et al., 2000a; Hanakawa et al., 2009). The finding that both sub- and suprathreshold rTMS of M1 can be accompanied by activation changes in remotely located regions was further corroborated by the identification of BOLD increases in cortical as well as subcortical motor structures that largely corresponded to the network of activations found during actual finger movements in the absence of TMS (Bestmann et al., 2004; Denslow et al., 2005). Furthermore, remote activation changes were also found beyond the time window of stimulation when applying an offline $10 \mathrm{~Hz}$ rTMS protocol to right M1 between two scanning sessions, with enhanced activations in the motor network nodes accompanied by faster motor performance (Yoo et al., 2008; sensory network nodes activated by noxious stimulation on the other hand showed post-rTMS reductions in activation level). Overall, these findings seem to indicate that M1TMS readily induces activation changes detectable by BOLD fMRI in remote motor regions, whereas local effects immediately under the coil are more elusive. This conclusion is in line with some of the EEG studies discussed earlier, in which $5 \mathrm{~Hz}$ rTMS to M1 led to potentiated responses in premotor cortex, but not in M1 itself (Esser et al., 2006; Huber et al., 2007). Based on modeling work (Esser et al., 2005), these authors suggest that local TMS-induced activity changes are less apparent because both excitatory and inhibitory neural populations are affected here (such that the net activation is weaker and more difficult to detect), whereas longerrange connections mainly constitute excitatory pathways leading to stronger and longer-lasting remote responses that are more easily observed. However, the currently still ill-defined intricacies of TMS-induced effects on local neural circuitry and the additional translation to haemodynamic signals (which might partially explain why BOLD responses are more readily observed in remote regions due to the fact that the fMRI signal more closely reflects incoming and intracortical processing than spiking output; Logothetis, 2008) continue to stand in the way of clear-cut interpretations (see Logothetis et al., 2010 for an elegant demonstration of how local neural circuitry dictates the propagation of externally induced stimulation as observed using fMRI).

\subsubsection{Target site: premotor cortex}

Using two subthreshold intensities in terms of overt motor output, Bestmann et al. (2005) showed that $10 \mathrm{~s}$ of $3 \mathrm{~Hz}$ rTMS over left dorsal premotor cortex (PMd) led to positive BOLD responses in connected motor regions such as the right PMd, bilateral ventral premotor cortex, supplementary and cingulate motor areas, cerebellum and caudate nucleus. These regions largely overlapped with the network activated during the actual performance of a finger tapping task (although it should be noted that no TMS effect was elicited in the ipsilateral M1). With the higher stimulation intensity, signal increases could also be observed at the target site itself (see also Bestmann et al., 2008b). Therefore, previous reports on the lack of local responsiveness in non-primary motor areas (Baudewig et al., 2001; Kemna and Gembris, 2003) might mainly reflect the point that the applied stimulation was not frequent or long enough since the intensities were chosen relative to the threshold defined in M1 whereas other areas might have higher excitability thresholds.

Another important finding based on left PMd-TMS was that the elicited remote activations can show both state- and taskdependent effects. Bestmann et al. (2008b) found that the right PMd and M1 showed activation increases and stronger functional coupling following TMS (when comparing high vs. low intensity stimulation) while subjects were engaged in a grip force task using their left hand. Conversely, the identical contrast showed activation decreases in these areas when applying TMS at rest in the same subjects. Similarly, O'Shea et al. (2007) reported compensatory activation increases in the contralateral right PMd (and related medial premotor areas) following $15 \mathrm{~min}$ of offline $1 \mathrm{~Hz}$ TMS over left PMd. Importantly, this stronger involvement of 
the right PMd was only observed during a motor task that would normally also heavily engage left PMd (namely an action selection task), and not during a more simple action execution task (repeatedly performing the same finger movement). Moreover, this post-TMS increase in the contralateral PMd proved to be causally relevant as a functional compensatory mechanism to retain task performance. This became evident as behavioral performance suffered when applying dpTMS to interfere with the compensatory right PMd activation following the $1 \mathrm{~Hz}$ rTMS treatment of the left PMd.

\subsubsection{Target sites: frontal eye fields $\mathcal{E}$ parietal cortex}

In a series of publications, Ruff et al. (2006, 2008, 2009b) report the effect of short 5-pulse TMS trains at $9 \mathrm{~Hz}$ and different intensities applied to either the right or left FEF and IPS. Subjects were instructed to passively fixate a central fixation point while TMS was applied either during rest or during visual stimulation by means of bilaterally presented flickering checkerboards. The main results can be summarized as follows: high-intensity right FEF TMS led to bilateral activity increases in the subregions of early visual cortex (V1 to V4) representing the peripheral visual field, and decreases in the subregions coding for the central visual field. These remote effects were observed irrespective of whether TMS was applied at rest or during concurrent visual stimulation. Interestingly, an additional psychophysical experiment confirmed that TMS to this frontal site selectively enhanced the perceived contrast of peripherally presented visual stimuli. Although left FEF TMS elicited similar BOLD decreases for central visual field representations in early visual cortex, no changes were found for the peripheral parts of the visual scene. Neither left nor right FEF TMS had an effect on motion-selective area V5/MT. In contrast, applying TMS to the right IPS decreased the BOLD response in V5/ MT (but only when a moving visual stimulus was simultaneously presented), yet resulted in activation increases throughout areas V1-V4 only when no visual stimulus was shown (irrespective of which cortical eccentricity representation was considered). Finally, left IPS TMS did not have any observable effects on the activity level of the studied visual brain areas. In sum, these findings underline the state-dependence of TMS effects and provide another illustration of how changing the stimulation site can reveal different types of remote responses.

The right FEF was also targeted in a study applying theta burst rTMS, revealing that TBS suppressed local BOLD activity in a saccade task 20-35 min after, but not immediately following rTMS (Hubl et al., 2008). Additional, yet weaker, suppression was also apparent in remote regions such as the (pre)supplementary and parietal eye fields.

Analogous to the previously discussed data on PMd-TMS (Bestmann et al., 2008b), Blankenburg et al. (2008) could show that right parietal TMS can enhance processing in the left primary somatosensory cortex in the presence of concomitant somatosensory input via median nerve stimulation, while conversely suppressing activity when peripheral input is absent. In parallel, bilateral responses in a predefined thalamic ROI were strongest when TMS and peripheral somatosensory stimulation were paired, suggesting that under normal conditions the cortex is able to interhemispherically modulate thalamic sensory gating functions. Again, separately gathered behavioral data confirmed that the used TMS protocol resulted in improvements in detecting perithreshold median nerve stimulation, suggesting that the observed fMRI signal increases were functionally relevant.

Complementary to the 'bottom-up'-driven state dependency discussed so far (Blankenburg et al., 2008; Ruff et al., 2008), parietal TMS can also have a differential impact on brain states that are controlled in a more 'top down' manner. In an experiment by Sack et al. (2007), subjects either performed an angle or color discrimination task based on identical images of an analogue clock's hands. Interestingly, short TMS trains only led to BOLD signal decreases in the stimulated right SPL, and more distal postcentral and middle frontal gyrus when subjects executed the angle task. Other areas, such as the bilateral SMA and right FEF showed similar TMS-induced BOLD decreases irrespective of which task was performed. Importantly, the task-specific BOLD reductions following TMS correlated with the induced behavioral impairment: the stronger the reduction, the slower subjects responded. Furthermore, TMS to the left SPL did not result in such modulations.

Another example of the influence TMS can have on more internally generated brain states comes from a study in which $1 \mathrm{~Hz}$ rTMS over left SPL was administered $10 \mathrm{~s}$ before subjects either executed or imagined movements involving their wrist (de Vries et al., 2009). TMS resulted in increased activations in bilateral prefrontal, right temporo-parietal and left posterior parietal cortex (during movement execution) and left medial prefrontal cortex, supramarginal gyrus and right lateral prefrontal and posterior parietal cortex (during motor imagery), interpreted as compensatory activations to cope with the reduced ability to estimate the proprioceptive consequences of the prepared movement following TMS.

\subsubsection{Target sites: prefrontal cortex and primary sensory cortex (SI)}

The only study in which the left PFC was targeted used $21 \mathrm{~s}$ of $1 \mathrm{~Hz}$ rTMS at 80,100 or $120 \%$ of the rMT during rest (Nahas et al., 2001). Beyond the consistently reported activation of auditory cortex that was present in all three conditions, TMS at the lowest intensity did not produce any additional activations (though it should be noted that whole brain coverage was not achieved). Conversely, the highest intensity led to bilateral prefrontal activation, so including the stimulation site. This finding is in accordance with the notion that local BOLD responses in brain areas outside of M1 can be elicited when going beyond the motor threshold intensity (which apparently represents an overestimation of the excitability of other cortical regions based on the responses evoked in the more readily excited motor cortex).

Also offline rTMS can modulate responses locally, as shown for left primary somatosensory cortex following $5 \mathrm{~Hz}$ stimulation by Pleger et al. (2006). Post-rTMS activations during a tactile frequency discrimination task were both enlarged and enhanced compared to sham stimulation. In addition, this rTMS-induced BOLD signal change was positively correlated with the gain in discrimination ability after TMS. Contrastingly, an activation cluster within M1 correlated negatively with these behavioral improvements following TMS, and was also negatively related with the activation level found for SI. This finding was later corroborated via dynamic causal modeling, pointing at local increases in SI excitability and concomitant increases in the effective connectivity between SI and M1. Moreover, these changes were no longer observable $2 \mathrm{~h}$ post-rTMS and did also not emerge when performing rTMS over left SI and subsequently performing the tactile frequency discrimination task with the ipsilateral left index finger, emphasizing the specificity of the obtained results.

\subsubsection{Summary TMS-fMRI}

The reviewed TMS-fMRI papers further substantiate the notion that TMS to a particular cortical target site does not solely produce focal effects by selectively affecting an isolated patch of cortex. Rather, target sites should be considered as nodes within a widespread network of interacting brain regions, where perturbing or boosting processing of one element can also influence several others. Moreover, the current state of such a neural network, either induced by incoming sensory input or intrinsically set to meet current task demands, determines the impact of TMS and the 
associated network dynamics. Interestingly, several experiments showed that the evoked BOLD signal changes were correlated with changes in task performance, underlining the functional relevance of the observed modulations in fMRI activation patterns. These brain-behavior associations have been derived in two complementary ways. Remote TMS effects observed using combined TMSfMRI have led to predictions on behavioral consequences that were subsequently tested in separate psychophysical experiments (demonstrating how multimodal TMS research can generate new hypotheses; e.g., Ruff et al., 2006). Alternatively, TMS has been applied during the execution of a particular task, while concurrently documenting the TMS network effects that underlie the observed behavioral change using fMRI (e.g., Sack et al., 2007). Whether the net effect on performance was positive (Blankenburg et al., 2008; Pleger et al., 2006; Ruff et al., 2006) or negative (Sack et al., 2007) depended on the chosen target site and the task at hand. A caveat to be kept in mind in this context is that although correlations between TMS-related behavioral changes and observed remote activation changes might be interpreted as convincing evidence for the functional relevance of the identified remote area, such conclusions are in fact again hampered by the inability to infer causality from correlational measures. In other words, such interpretations on behavioral impact are better tested independently by applying TMS to the identified remote network node. For example, in a follow-up of the previously mentioned study by Sack et al. (2007), the initially observed correlation between the remote activation change in the middle frontal gyrus and the behavioral impairments in spatial task performance was explicitly tested. By localizing the middle frontal gyrus through fMRI functional connectivity analyses and subsequently targeting the same area with TMS, causal evidence was provided for the functional relevance of the remote TMS activation change identified earlier (de Graaf et al., 2009). Only such an iterative approach can directly verify the functional role of revealed response profiles in distant network nodes.

\section{Discussion and future perspectives}

The presented review provides an overview of the current state of affairs in the domain of multimodal TMS research. It describes the main experimental approaches and core findings for the most frequently investigated TMS target sites in studies combining TMS with either EEG, PET or fMRI. The ability to concurrently record activation changes on the neural systems level is of paramount importance to better grasp the direct impact of any given TMS protocol without having to rely on behavioral outcome measures alone. More specifically, the simultaneous use of TMS and different neuroimaging techniques allows to characterize both local (i.e., in the cortical tissue directly under the TMS coil) and remote (i.e., distant from the original cortical target site) effects of TMS in more detail, leading to a better understanding of TMS-induced modulations in neural processing and cognitive task performance (Sack, 2010).

Irrespective of the details of any of the discussed studies or modalities, there are a number of relevant general conclusions one can draw based on the existing multimodal literature.

First, the distribution across studies regarding the chosen target site and experimental conditions is heavily skewed. The large majority of studies has focused on primary motor cortex, leading to a relative underrepresentation of other cortical regions as potential TMS target sites. This bias towards M1 is most likely due to the ease with which overtly observable responses (namely MEPs) can be evoked here, such that coil positioning and independent verification of TMS effects are more straightforward than for any other target site. In addition, a lot of unimodal TMS studies have successfully targeted M1 for the same reasons, providing a wealth of background information to build on. Similarly, especially in the TMS-PET literature, there are far more reports on the effects of TMS while participants are in an awake resting state, compared to situations in which they are actively engaged in a task. Again, this is a reasonable choice as resting state modulations constitute a good starting point, whereas investigating interactions between task instructions and TMS undoubtedly adds another level of complexity. The downside, however, is that we have only scratched the surface of exploring the neural effects of TMS during cognitive task performance and their relation to behavioral outcome measures.

Second, with all three modalities, local effects of TMS have been measured in the direct vicinity of the coil. This is maybe not too surprising given the multitude of unimodal TMS papers showing consistent behavioral effects of TMS that are specific to a particular target site. However, in this light it is interesting to note that some fMRI studies have failed to visualize these effects with subthreshold TMS intensities, whereas EEG appears to be the most sensitive method to detect TMS-induced modulations.

Third, there is overwhelming evidence for remote effects of TMS, a finding that is very consistent across modalities. Importantly, distant evoked responses are exclusively found in areas that are either anatomically or functionally connected to the TMS target site, arguing against a non-specific (water ripple-like) spread of neural activity throughout adjacent cortical tissue. This holds for cortical and subcortical structures alike. In addition, the revealed networks (often appearing following TMS during rest) have been found to be quite similar to task-related networks involving the stimulated site by direct comparison to task execution conditions within the same experiment.

Fourth, these remote TMS effects have been shown to be statedependent in the sense that the brain's current state, dictated by ongoing task demands or simply modulated by external sensory stimulation or naturally occurring fluctuations, can influence the response to TMS. Again, demonstrations of this state-dependence can be found in all three neuroimaging modalities. Interestingly, these findings suggest that TMS-induced neural activity is particularly likely to spread to nodes within a (currently active) functional network, and does not necessarily spread indifferently to any of the regions anatomically connected to the target site. Whereas some of the discussed EEG studies show that single TMS pulses indeed seem to travel more easily through already activated networks, there are fMRI findings indicating that the direction of remote effects (activation increases/decreases) can actually be reversed depending on the system's state. Such variations are likely to reflect differences in the initial brain states themselves, related to interregional mutual inhibition or facilitation mechanisms. In any case, state-dependent modulations of TMS effects should not be underestimated and could also partially explain differences in remote effects between target sites even though the same TMS protocol is used. For example, compared to 'higher order' cognitive areas such as prefrontal cortex, the 'resting state' in M1 might be much more homogeneous across subjects, leading to more consistent TMS-induced activation changes (see also Speer et al., 2003a,b). One general caveat that might be particularly relevant for task-induced statedependent effects concerns the difficulty of teasing apart whether the obtained neuroimaging results truly reveal the underlying network connectivity or should more accurately be interpreted as indications of the way in which the brain is trying to cope with the artificial disturbance by TMS while trying to maintain proper task execution (Sack et al., 2005). Even if no changes in behavioral performance are observed following TMS, this does not mean that no compensatory adjustments have taken place. On the other hand, one should note that this issue holds for any interference technique and therefore is not unique to TMS. 
Finally, when looking at the different parameters than can be varied when applying TMS, variations in stimulation intensity most consistently alter both local and remote response amplitudes, without changing the layout of the revealed network or the associated response latencies. The picture is less clear when varying stimulation frequency (let alone second-order frequencies related to the time interval between trains of stimulation), defying simple dichotomies in terms of clear cut-off points between lowand high-frequency TMS, respectively, assumed to result in inhibitory or facilitatory modulations following repetitive stimulation. Conversely, evidence of prolonged (i.e., outlasting the period of stimulation) neural activity changes following conventional repetitive or theta-burst stimulation has been obtained using all of the reviewed neuroimaging modalities.

Although interesting insights have been gathered based on the wide range of findings reviewed here, how could some of the revealed inconsistencies be resolved in future studies?

Foremost, more basic research is required exploiting the already existing multimodal imaging facilities by systematically comparing the recorded neural responses to different TMS parameter settings. Similarly, the consequences of identical variations in TMS settings should ideally be compared across different modalities, preferably within the same subject (or even simultaneously within the same session). Only in this way can multimodal results really be synthesized, both for currently already commonly used TMS protocols, as well as for all kinds of new protocols that are likely to even expand the varying types of influence TMS can have on the working human brain. Naturally, such efforts would also benefit greatly from additional work outside the TMS research field on how the signals obtained with EEG, PET and fMRI can actually be integrated and might differently reflect an identical neural event (e.g., Laufs, 2008; Logothetis, 2003; Ritter and Villringer, 2006).

Secondly, it will become imperative to scrutinize variations of TMS-induced neural activation patterns both across trials at the within-subject level (e.g., through pre-TMS fluctuations in relevant EEG frequency ranges) and across individual subjects (e.g., Hamidi et al., 2009), instead of trying to capture these effects by pooled group results only (e.g., see Ridding and Ziemann, 2010 for a diverse range of factors influencing TMS-induced cortical plasticity).

Moreover, studies employing other complementary techniques such as MR spectroscopy (Stagg et al., 2009), functional nearinfrared spectroscopy (Hada et al., 2006; Kozel et al., 2009; Mochizuki et al., 2006), and diffusion weighted imaging (DWI) of white matter bundles (Klöppel et al., 2008) will further extend the types of questions that can be answered. An illustrative example, which also touches upon the previous point, comes from a study in which individual differences in micro-scale white matter structure (as measured by DWI) were found to be correlated with differences in functional connectivity indexed by TMS (Boorman et al., 2007).

Finally, the neural system's level viewpoint outlined here will have to rely on continued studies at a more fine-grained analysis level through invasive animal research (e.g., see Funke and Benali, 2010), to be able to understand which neurophysiological mechanisms are really affected by TMS and how these effects in turn spread to remote sites and ultimately may alter behavior. Some very important contributions have already come from work primarily performed in cats (Allen et al., 2007; Aydin-Abidin et al., 2006; de Labra et al., 2007; Moliadze et al., 2005, 2003; Pasley et al., 2009; Valero-Cabré et al., 2005, 2007), but also rodents (AydinAbidin et al., 2008; Trippe et al., 2009) and monkeys (Hayashi et al., 2004; Ohnishi et al., 2004b). However, due to intrinsic technical constraints of several of the employed invasive methods used so far, remote TMS effects have not yet been intensively investigated in animal models. Moreover, due to the already mentioned intrinsic intricacies of neural circuits, a multimodal approach by concurrent use of complementary methods will also be required in this line of research (Logothetis, 2008).

By actively pursuing all of these research avenues, the role of TMS as a valuable and versatile neuroscientific research tool will be further substantiated and extended. We have reached a new era in which the flexible use of multimodal research facilities allows us to directly monitor the TMS-induced changes in brain activity at the neural network level, providing unique insights into the causal relations within and between large scale circuits of the human brain in vivo. Ultimately, a better understanding of the neural effects of TMS obtained by multimodal imaging will hopefully also lead to a better informed translation to clinical applications, resulting in more effective and well-controlled therapeutic interventions.

\section{Acknowledgements}

This work was supported by grant \#452-06-003 from the Netherlands Organization of Scientific Research (NWO) to ATS.

\section{Appendix A. Supplementary data}

Supplementary data associated with this article can be found, in the online version, at doi:10.1016/j.pneurobio.2011.04.004.

\section{References}

Abraham, W.C., Bear, M.F., 1996. Metaplasticity: the plasticity of synaptic plasticity. Trends Neurosci. 19, 126-130.

Allen, E.A., Pasley, B.N., Duong, T., Freeman, R.D., 2007. Transcranial magnetic stimulation elicits coupled neural and hemodynamic consequences. Science 317 (5846), 1918-1921.

Amassian, V.E., Cracco, R.Q., Maccabee, P.J., Cracco, J.B., 1992. Cerebello-frontal cortical projections in humans studied with the magnetic coil. Electroencephalogr. Clin. Neurophysiol. 85 (4), 265-272.

Aydin-Abidin, S., Moliadze, V., Eysel, U.T., Funke, K., 2006. Effects of repetitive TMS on visually evoked potentials and EEG in the anaesthetized cat: dependence on stimulus frequency and train duration. J. Physiol. 574 (Pt 2), 443-455.

Aydin-Abidin, S., Trippe, J., Funke, K., Eysel, U.T., Benali, A., 2008. High- and lowfrequency repetitive transcranial magnetic stimulation differentially activates c-Fos and zif268 protein expression in the rat brain. Exp. Brain Res. 188 (2), 249-261.

Barker, A., Jalinous, R., Freeston, I.L., 1985. Non-invasive magnetic stimulation of human motor cortex. The Lancet 1 (8437), 1106-1107.

Barr, M.S., Farzan, F., Rusjan, P.M., Chen, R., Fitzgerald, P.B., Daskalakis, Z.J., 2009. Potentiation of gamma oscillatory activity through repetitive transcranial magnetic stimulation of the dorsolateral prefrontal cortex. Neuropsychopharmacology 34 (11), 2359-2367.

Baudewig, J., Siebner, H.R., Bestmann, S., Tergau, F., Tings, T., Paulus, W., Frahms, J., 2001. Functional MRI of cortical activations induced by transcranial magnetic stimulation (TMS). Neuroreport 12 (16), 3543-3548.

Bestmann, S., Baudewig, J., Siebner, H.R., Rothwell, J.C., Frahm, J., 2003. Subthreshold high-frequency TMS of human primary motor cortex modulates interconnected frontal motor areas as detected by interleaved fMRI-TMS. Neuroimage 20 (3), 1685-1696.

Bestmann, S., Baudewig, J., Siebner, H.R., Rothwell, J.C., Frahm, J., 2004. Functional MRI of the immediate impact of transcranial magnetic stimulation on cortical and subcortical motor circuits. Eur. J. Neurosci. 19 (7), 1950-1962.

Bestmann, S., Baudewig, J., Siebner, H.R., Rothwell, J.C., Frahm, J., 2005. BOLD MRI responses to repetitive TMS over human dorsal premotor cortex. Neuroimage 28 (1), 22-29.

Bestmann, S., Ruff, C.C., Blankenburg, F., Weiskopf, N., Driver, J., Rothwell, J.C., 2008a. Mapping causal interregional influences with concurrent TMS-fMRI. Exp. Brain Res. 191 (4), 383-402.

Bestmann, S., Swayne, O., Blankenburg, F., Ruff, C.C., Haggard, P., Weiskopf, N., Josephs, O., Driver, J., Rothwell, J.C., Ward, N.S., 2008b. Dorsal premotor cortex exerts state-dependent causal influences on activity in contralateral primary motor and dorsal premotor cortex. Cereb. Cortex 18 (6), 1281-1291.

Bikmullina, R., Kicić, D., Carlson, S., Nikulin, V.V., 2009. Electrophysiological correlates of short-latency afferent inhibition: a combined EEG and TMS study. Exp. Brain Res. 194 (4), 517-526.

Blankenburg, F., Ruff, C.C., Bestmann, S., Bjoertomt, O., Eshel, N., Josephs, O., Weiskopf, N., Driver, J., 2008. Interhemispheric effect of parietal TMS on somatosensory response confirmed directly with concurrent TMS-fMRI. J. Neurosci. 28 (49), 13202-13208. 
Bliss, T.V.P., Lømo, T., 1973. Long-lasting potentiation of synaptic transmission in the dentate area of the anaesthetized rabbit following stimulation of the perforant path. J. Physiol. 232, 331-356.

Bohning, D.E., Shastri, A., Nahas, Z., Lorberbaum, J.P., Andersen, S.W., Dannels, W.R., Haxthausen, E., Vincent, D.J., George, M.S., 1998. Echoplanar BOLD fMRI of brain activation induced by concurrent transcranial magnetic stimulation. Invest. Radiol. 33, 336-340.

Bohning, D.E., Shastri, A., McConnell, K.A., Nahas, Z., Lorberbaum, J.P., Roberts, D.R., Teneback, C., Vincent, D.J., George, M.S., 1999. A combined TMS/fMRI study of intensity-dependent TMS over motor cortex. Biol. Psychiatry 45, 385-394.

Bohning, D.E., Shastri, A., Wassermann, E.M., Ziemann, U., Lorberbaum, J.P., Nahas, Z., Lomarev, M.P., George, M.S., 2000a. BOLD-fMRI response to single-pulse transcranial magnetic stimulation (TMS). J. Magn. Reson. Imaging 11 (6), 569-574.

Bohning, D.E., Shastri, A., McGavin, L., McConnell, K.A., Nahas, Z., Lorberbaum, J.P., Roberts, D.R., George, M.S., 2000b. Motor cortex brain activity induced by $1-\mathrm{Hz}$ transcranial magnetic stimulation is similar in location and level to that for volitional movement. Invest. Radiol. 35 (11), 676-683.

Bohning, D.E., Shastri, A., Lomarev, M.P., Lorberbaum, J.P., Nahas, Z., George, M.S., 2003. BOLD-fMRI response vs. transcranial magnetic stimulation (TMS) pulsetrain length: testing for linearity. J. Magn. Reson. Imaging 17 (3), 279-290.

Bonato, C., Miniussi, C., Rossini, P.M., 2006. Transcranial magnetic stimulation and cortical evoked potentials: a TMS/EEG co-registration study. Clin. Neurophysiol. 117 (8), 1699-1707.

Boorman, E.D., O’Shea, J., Sebastian, C., Rushworth, M.F.S., Johansen-Berg, H., 2007. Individual differences in white-matter microstructure reflect variation in functional connectivity during choice. Curr. Biol. 17 (16), 1426-1431.

Brignani, D., Manganotti, P., Rossini, P.M., Miniussi, C., 2008. Modulation of cortical oscillatory activity during transcranial magnetic stimulation. Hum. Brain Mapp. 29 (5), 603-612.

Capotosto, P., Babiloni, C., Romani, G.L., Corbetta, M., 2009. Frontoparietal cortex controls spatial attention through modulation of anticipatory alpha rhythms. J. Neurosci. 29 (18), 5863-5872.

Cheeran, B., Koch, G., Stagg, C.J., Baig, F., Teo, J., 2010. Transcranial Magnetic Stimulation: from neurophysiology to pharmacology, molecular biology and genomics. Neuroscientist 16 (3), 210-221

Chen, W., Mima, T., Siebner, H.R., Oga, T., Hara, H., Satow, T., Begum, T., Nagamine, T. Shibasaki, H., 2003. Low-frequency rTMS over lateral premotor cortex induces lasting changes in regional activation and functional coupling of cortical motor areas. Clin. Neurophysiol. 114 (9), 1628-1637.

Cho, S.S., Strafella, A.P., 2009. rTMS of the left dorsolateral prefrontal cortex modulates dopamine release in the ipsilateral anterior cingulate cortex and orbitofrontal cortex. PloS One 4 (8), e6725.

Chouinard, P.A., van der Werf, Y.D., Leonard, G., Paus, T., 2003. Modulating neural networks with transcranial magnetic stimulation applied over the dorsal premotor and primary motor cortices. J. Neurophysiol. 90 (2), 1071-1083.

Cracco, R.Q., Amassian, V.E., Maccabee, P.J., Cracco, J.B., 1989. Comparison of human transcallosal responses evoked by magnetic coil and electrical stimulation. Electroencephalogr. Clin. Neurophysiol. 74 (6), 417-424.

Daskalakis, Z.J., Farzan, F., Barr, M.S., Maller, J.J., Chen, R., Fitzgerald, P.B., 2008. Longinterval cortical inhibition from the dorsolateral prefrontal cortex: a TMS-EEG study. Neuropsychopharmacology 33 (12), 2860-2869.

Davare, M., Rothwell, J.C., Lemon, R.N., 2010. Causal connectivity between the human anterior intraparietal area and premotor cortex during grasp. Curr. Biol. 20 (2), 176-181.

De Gennaro, L., Fratello, F., Marzano, C., Moroni, F., Curcio, G., Tempesta, D. Pellicciari, M.C., Pirulli, C., Ferrara, M., Rossini, P.M., 2008. Cortical plasticity induced by transcranial magnetic stimulation during wakefulness affects electroencephalogram activity during sleep. PloS One 3 (6), e2483.

de Labra, C., Rivadulla, C., Grieve, K., Mariño, J., Espinosa, N., Cudeiro, J., 2007. Changes in visual responses in the feline dLGN: selective thalamic suppression induced by transcranial magnetic stimulation of V1. Cereb. Cortex 17 (6), 13761385.

Denslow, S., Lomarev, M.P., George, M.S., Bohning, D.E., 2005. Cortical and subcortical brain effects of transcranial magnetic stimulation (TMS)-induced movement: an interleaved TMS/functional magnetic resonance imaging study. Biol. Psychiatry 57 (7), 752-760.

de Vries, P.M., de Jong, B.M., Bohning, D.E., Walker, J.A., George, M.S., Leenders, K.L., 2009. Changes in cerebral activations during movement execution and imagery after parietal cortex TMS interleaved with 3T MRI. Brain Res. 1285, 58-68.

Driver, J., Blankenburg, F., Bestmann, S., Vanduffel, W., Ruff, C.C., 2009. Concurrent brain-stimulation and neuroimaging for studies of cognition. Trends Cogn. Sci. 13 (7), 319-327.

Eisenegger, C., Treyer, V., Fehr, E., Knoch, D., 2008. Time-course of "off-line" prefrontal rTMS effects-a PET study. Neuroimage 42 (1), 379-384.

Enomoto, H., Ugawa, Y., Hanajima, R., Yuasa, K., Mochizuki, H., Terao, Y., Shiio, Y., Furubayashi, T., Iwata, N.K., Kanazawa, I., 2001. Decreased sensory cortical excitability after $1 \mathrm{~Hz}$ rTMS over the ipsilateral primary motor cortex. Clin. Neurophysiol. 112 (11), 2154-2158.

Esser, S.K., Hill, S.L., Tononi, G., 2005. Modeling the effects of transcranial magnetic stimulation on cortical circuits. J. Neurophysiol. 94 (1), 622-639.

Esser, S.K., Huber, R., Massimini, M., Peterson, M.J., Ferrarelli, F., Tononi, G., 2006. A direct demonstration of cortical LTP in humans: a combined TMS/EEG study. Brain Res. Bull. 69 (1), 86-94.

Evers, S., Böckermann, I., Nyhuis, P.W., 2001. The impact of transcranial magnetic stimulation on cognitive processing: an event-related potential study. Neuroreport 12 (13), 2915-2918.
Farzan, F., Barr, M.S., Wong, W., Chen, R., Fitzgerald, P.B., Daskalakis, Z.J., 2009 Suppression of $\gamma$-oscillations in the dorsolateral prefrontal cortex following long interval cortical inhibition: a TMS-EEG study. Neuropsychopharmacology 34, 1543-1551.

Ferrarelli, F., Haraldsson, H.M., Barnhart, T.E., Roberts, A.D., Oakes, T.R., Massimini, M., Stone, C.K., Kalin, N.H., Tononi, G., 2004. A [17F]-fluoromethane PET/TMS study of effective connectivity. Brain Res. Bull. 64 (2), 103-113.

Fitzgerald, P.B., Maller, J.J., Hoy, K., Farzan, F., Daskalakis, Z.J., 2009. GABA and cortical inhibition in motor and non-motor regions using combined TMS-EEG: a time analysis. Clin. Neurophysiol. 120 (9), 1706-1710.

Fox, P.T., Ingham, R., George, M.S., Mayberg, H., Ingham, J., Roby, J., Martin, C., Jerabek, P., 1997. Imaging human intra-cerebral connectivity by PET during TMS. Neuroreport 8 (12), 2787-2791.

Fox, P.T., Narayana, S., Tandon, N., Fox, S.P., Sandoval, H., Kochunov, P., Capaday, C., Lancaster, J.L., 2006. Intensity modulation of TMS-induced cortical excitation: primary motor cortex. Hum. Brain Mapp. 27 (6), 478-487.

Fuggetta, G., Fiaschi, A., Manganotti, P., 2005. Modulation of cortical oscillatory activities induced by varying single-pulse transcranial magnetic stimulation intensity over the left primary motor area: a combined EEG and TMS study. Neuroimage 27 (4), 896-908.

Fuggetta, G., Pavone, E.F., Walsh, V., Kiss, M., Eimer, M., 2006. Cortico-cortical interactions in spatial attention: a combined ERP/TMS study. J. Neurophysiol. 95 (5), 3277-3280.

Fuggetta, G., Pavone, E.F., Fiaschi, A., Manganotti, P., 2008. Acute modulation of cortical oscillatory activities during short trains of high-frequency repetitive transcranial magnetic stimulation of the human motor cortex: a combined EEG and TMS study. Hum. Brain Mapp. 29 (1), 1-13.

Fumal, A., Bohotin, V., Vandenheede, M., Seidel, L., de Pasqua, V., Maertens de Noordhout, A., Schoenen, J., 2003. Effects of repetitive transcranial magnetic stimulation on visual evoked potentials: new insights in healthy subjects. Exp. Brain Res. 150 (3), 332-340.

Fumal, A., Coppola, G., Bohotin, V., Gérardy, P.-Y., Seidel, L., Donneau, A.-F., Vandenheede, M., Maertens de Noordhout, A., Schoenen, J., 2006. Induction of longlasting changes of visual cortex excitability by five daily sessions of repetitive transcranial magnetic stimulation (rTMS) in healthy volunteers and migraine patients. Cephalalgia 26 (2), 143-149.

Funke, K., Benali, A., 2010. Cortical cellular actions of transcranial magnetic stimulation. Restor. Neurol. Neurosci. 28 (4), 399-417.

Graaf, T., de, Jacobs, C., Roebroeck, A., Sack, A.T., 2009. FMRI effective connectivity and TMS chronometry: complementary accounts of causality in the visuospatial judgment network. PloS One 4 (12), e8307.

Griskova, I., Ruksenas, O., Dapsys, K., Herpertz, S., Höppner, J., 2007. The effects of $10 \mathrm{~Hz}$ repetitive transcranial magnetic stimulation on resting EEG power spectrum in healthy subjects. Neurosci. Lett. 419 (2), 162-167.

Grossheinrich, N., Rau, A., Pogarell, O., Hennig-Fast, K., Reinl, M., Karch, S., Dieler, A., Leicht, G., Mulert, C., Sterr, A., Padberg, F., 2009. Theta burst stimulation of the prefrontal cortex: safety and impact on cognition, mood, and resting electroencephalogram. Biol. Psychiatry 65 (9), 778-784.

Hada, Y., Abo, M., Kaminaga, T., Mikami, M., 2006. Detection of cerebral blood flow changes during repetitive transcranial magnetic stimulation by recording hemoglobin in the brain cortex, just beneath the stimulation coil, with nearinfrared spectroscopy. Neuroimage 32 (3), 1226-1230.

Hamidi, M., Slagter, H.A., Tononi, G., Postle, B.R., 2009. Repetitive transcranial magnetic stimulation affects behavior by biasing endogenous cortical oscillations. Front. Integr. Neurosci. 3, 1-12.

Hanakawa, T., Mima, Tatsuya, Matsumoto, R., Abe, M., Inouchi, M., Urayama, S.-I., Anami, K., Honda, M., Fukuyama, H., 2009. Stimulus-response profile during single-pulse transcranial magnetic stimulation to the primary motor cortex. Cereb. Cortex 19 (11), 2605-2615.

Hansenne, M., Laloyaux, O., Mardaga, S., Ansseau, M., 2004. Impact of low frequency transcranial magnetic stimulation on event-related brain potentials. Biol. Psychol. 67 (3), 331-341.

Hayashi, T., Ohnishi, T., Okabe, Shingo, Teramoto, N., Nonaka, Y., Watabe, H., Imabayashi, E., Otha, Y., Jino, H., Ejima, N., Sawada, T., Iida, H., Matsuda, H., Ugawa, Y., 2004. Long-term effect of motor cortical repetitive transcranial magnetic stimulation. Ann. Neurol. 56 (1), 77-85.

Hayward, G., Mehta, M.A., Harmer, C., Spinks, T.J., Grasby, P.M., Goodwin, G.M., 2007. Exploring the physiological effects of double-cone coil TMS over the medial frontal cortex on the anterior cingulate cortex: an H2(15)O PET study. Eur. J. Neurosci. 25 (7), 2224-2233.

Hilgetag, C.C., Théoret, H., Pascual-Leone, A., 2001. Enhanced visual spatial attention ipsilateral to rTMS-induced "virtual lesions" of human parietal cortex. Nat. Neurosci. 4 (9), 953-957.

Holler, I., Siebner, H.R., Cunnington, R., Gerschlager, W., 2006. 5 Hz repetitive TMS increases anticipatory motor activity in the human cortex. Neurosci. Lett. 392 (3), 221-225.

Hoogendam, J.M., Ramakers, G.M.J., Di Lazzaro, V., 2010. Physiology of repetitive transcranial magnetic stimulation of the human brain. Brain Stimul. 3, 95-118.

Huang, Y.-Z., Edwards, M.J., Rounis, E., Bhatia, K.P., Rothwell, J.C., 2005. Theta burst stimulation of the human motor cortex. Neuron 45 (2), 201-206.

Huang, Y.-Z., Rothwell, J.C., Chen, R.-S., Lu, C.-S., Chuang, W.-L., 2011. The theoretical model of theta burst form of repetitive transcranial magnetic stimulation. Clin. Neurophysiol. 122 (5), 1011-1018.

Huber, R., Esser, S.K., Ferrarelli, F., Massimini, M., Peterson, M.J., Tononi, G., 2007. TMS-induced cortical potentiation during wakefulness locally increases slow wave activity during sleep. PloS One 2 (3), e276. 
Hubl, D., Nyffeler, T., Wurtz, P., Chaves, S., Pflugshaupt, T., Lüthi, M., von Wartburg, R., Wiest, R., Dierks, T., 2008. Time course of blood oxygenation level-dependent signal response after theta burst transcranial magnetic stimulation of the frontal eye field. Neuroscience 151 (3), 921-928.

Ilmoniemi, R.J., Virtanen, J., Ruohonen, J., Karhu, J., Aronen, H.J., Näätänen, R., Katila, T., 1997. Neuronal responses to magnetic stimulation reveal cortical reactivity and connectivity. Neuroreport 8 (16), 3537-3540.

Ilmoniemi, R.J., Kicić, D., 2010. Methodology for combined TMS and EEG. Brain Topogr. 22 (4), 233-248.

Ishikawa, S., Matsunaga, K., Nakanishi, R., Kawahira, K., Murayama, N., Tsuji, S., Huang, Y., Rothwell, J.C., 2007. Effect of theta burst stimulation over the human sensorimotor cortex on motor and somatosensory evoked potentials. Clin. Neurophysiol. 118 (5), 1033-1043.

Jing, H., Takigawa, M., Hamada, K., Okamura, H., Kawaika, Y., Yonezawa, T., Fukuzako, H., 2001a. Effects of high frequency repetitive transcranial magnetic stimulation on P(300) event-related potentials. Clin. Neurophysiol. 112 (2), 304-313.

Jing, H., Takigawa, M., Okamura, H., Doi, W., Fukuzako, H., 2001b. Comparisons of event-related potentials after repetitive transcranial magnetic stimulation. J. Neurol. 248 (3), 184-192.

Katayama, T., Rothwell, J.C., 2007. Modulation of somatosensory evoked potentials using transcranial magnetic intermittent theta burst stimulation. Clin. Neurophysiol. 118 (11), 2506-2511.

Kemna, L.J., Gembris, D., 2003. Repetitive transcranial magnetic stimulation induces different responses in different cortical areas: a functional magnetic resonance study in humans. Neurosci. Lett. 336, 85-88.

Kicić, D., Lioumis, P., Ilmoniemi, R.J., Nikulin, V.V., 2008. Bilateral changes in excitability of sensorimotor cortices during unilateral movement: combined electroencephalographic and transcranial magnetic stimulation study. Neuroscience 152 (4), 1119-1129.

Kimbrell, T.A., Dunn, R.T., George, M.S., Danielson, A.L., Willis, M.W., Repella, J.D., Benson, B.E., Herscovitch, P., Post, R.M., Wasserman, E.M., 2002. Left prefrontalrepetitive transcranial magnetic stimulation (rTMS) and regional cerebra glucose metabolism in normal volunteers. Psychiatry Res. 115 (3), 101-113.

Klimesch, W., Sauseng, P., Gerloff, C., 2003. Enhancing cognitive performance with repetitive transcranial magnetic stimulation at human individual alpha frequency. Eur. J. Neurosci. 17 (5), 1129-1133.

Klöppel, S., Bäumer, T., Kroeger, J., Koch, M.A., Büchel, C., Münchau, A., Siebner, H.R., 2008. The cortical motor threshold reflects microstructural properties of cerebral white matter. Neuroimage 40 (4), 1782-1791.

Knoch, D., Treyer, V., Regard, M., Müri, R., Buck, A., Weber, B., 2006. Lateralized and frequency-dependent effects of prefrontal rTMS on regional cerebral blood flow. Neuroimage 31 (2), 641-648.

Ko, J.H., Monchi, O., Ptito, A., Bloomfield, P., Houle, S., Strafella, A.P., 2008. Theta burst stimulation-induced inhibition of dorsolateral prefrontal cortex reveals hemispheric asymmetry in striatal dopamine release during a set-shifting task: a TMS-[(11)C]raclopride PET study. Eur. J. Neurosci. 28 (10), 2147-2155.

Kobayashi, M., Pascual-Leone, A., 2003. Transcranial magnetic stimulation in neurology. Lancet Neurol. 2 (3), 145-156.

Komssi, S., Aronen, H.J., Huttunen, J., Kesäniemi, M., Soinne, L., Nikouline, V.V., Ollikainen, M., Roine, R.O., Karhu, J., Savolainen, S., Ilmoniemi, R.J., 2002. Ipsiand contralateral EEG reactions to transcranial magnetic stimulation. Clin. Neurophysiol. 113 (2), 175-184

Komssi, S., Kähkönen, S., Ilmoniemi, R.J., 2004. The effect of stimulus intensity on brain responses evoked by transcranial magnetic stimulation. Hum. Brain Mapp. 21 (3), 154-164.

Komssi, S., Kähkönen, S., 2006. The novelty value of the combined use of electroencephalography and transcranial magnetic stimulation for neuroscience research. Brain Res. Rev. 52 (1), 183-192.

Komssi, S., Savolainen, P., Heiskala, J., Kähkönen, S., 2007. Excitation threshold of the motor cortex estimated with transcranial magnetic stimulation electroencephalography. Neuroreport 18 (1), 13-16.

Kozel, F.A., Tian, F., Dhamne, S., Croarkin, P.E., McClintock, S.M., Elliott, A., Mapes, K. Husain, S., Liu, M.M.H., 2009. Using simultaneous repetitive Transcranial Magnetic Stimulation/functional Near Infrared Spectroscopy (rTMS/fNIRS) to measure brain activation and connectivity. Neuroimage 47 (4), 1177-1184

Kujirai, T., Sato, M., Rothwell, J.C., Cohen, L.G., 1993. The effect of transcranial magnetic stimulation on median nerve somatosensory evoked potentials. Electroencephalogr. Clin. Neurophysiol. 89 (4), 227-234.

Kähkönen, S., Wilenius, J., Komssi, S., Ilmoniemi, R.J., 2004. Distinct differences in cortical reactivity of motor and prefrontal cortices to magnetic stimulation. Clin. Neurophysiol. 115 (3), 583-588.

Kähkönen, S., Komssi, S., Wilenius, J., Ilmoniemi, R.J., 2005. Prefrontal transcrania magnetic stimulation produces intensity-dependent EEG responses in humans. Neuroimage 24 (4), 955-960.

Laufs, H., 2008. Endogenous brain oscillations and related networks detected by surface EEG-combined fMRI. Hum. Brain Mapp. 29 (7), 762-769.

Lee, L., Siebner, H.R., Rowe, J.B., Rizzo, V., Rothwell, J.C., Frackowiak, R.S.J., Friston, K.J., 2003. Acute remapping within the motor system induced by low-frequenc repetitive transcranial magnetic stimulation. J. Neurosci. 23 (12), 5308-5318.

Lioumis, P., Kicić, D., Savolainen, P., Mäkelä, J.P., Kähkönen, S., 2009. Reproducibility of TMS-Evoked EEG responses. Hum. Brain Mapp. 30 (4), 1387-1396.

Litvak, V., Zeller, D., Oostenveld, R., Maris, E., Cohen, A., Schramm, A., Gentner, R. Zaaroor, M., Pratt, H., Classen, J., 2007. LTP-like changes induced by paired associative stimulation of the primary somatosensory cortex in humans: source analysis and associated changes in behaviour. Eur. J. Neurosci. 25 (9), 2862 2874.
Logothetis, N.K., 2003. The underpinnings of the BOLD functional magnetic resonance imaging signal. J. Neurosci. 23 (10), 3963-3971.

Logothetis, N.K., 2008. What we can do and what we cannot do with fMRI. Nature 453 (7197), 869-878.

Logothetis, N.K., Augath, M., Murayama, Y., Rauch, A., Sultan, F., Goense, J., Oeltermann, A., Merkle, H., 2010. The effects of electrical microstimulation on cortical signal propagation. Nat. Neurosci. 13 (10), 1283-1291.

Massimini, M., Ferrarelli, F., Huber, R., Esser, S.K., Singh, H., Tononi, G., 2005. Breakdown of cortical effective connectivity during sleep. Science 309 (5744), 2228-2232.

Massimini, M., Ferrarelli, F., Esser, S.K., Riednera, B., Huber, R., Murphy, M., Peterson, M.J., Tononi, G., 2007. Triggering sleep slow waves by transcranial magnetic stimulation. Proc. Natl. Acad. Sci. U.S.A. 104 (20), 8496-8501.

Miniussi, C., Cappa, S.F., Cohen, L.G., Floel, A., Fregni, F., Nitsche, M.A., Oliveri, M., Pascual-Leone, A., Paulus, W., Priori, A., Walsh, V., 2008. Efficacy of repetitive transcranial magnetic stimulation/transcranial direct current stimulation in cognitive neurorehabilitation. Brain Stimul. 1 (4), 326-336.

Miniussi, C., Thut, G., 2009. Combining TMS and EEG offers new prospects in cognitive neuroscience. Brain Topogr. 22 (4), 249-256.

Mochizuki, H., Ugawa, Y., Terao, Yasuo, Sakai, K.L., 2006. Cortical hemoglobinconcentration changes under the coil induced by single-pulse TMS in humans: a simultaneous recording with near-infrared spectroscopy. Exp. Brain Res. 169 (3), 302-310.

Moliadze, V., Zhao, Y., Eysel, U.T., Funke, K., 2003. Effect of transcranial magnetic stimulation on single-unit activity in the cat primary visual cortex. J. Physiol. 553 (Pt 2), 665-679.

Moliadze, V., Giannikopoulos, D., Eysel, U.T., Funke, K., 2005. Paired-pulse transcranial magnetic stimulation protocol applied to visual cortex of anaesthetized cat: effects on visually evoked single-unit activity. Physiology 566 (Pt 3), 955-965.

Morishima, Y., Akaishi, R., Yamada, Y., Okuda, J., Toma, K., Sakai, K., 2009. Taskspecific signal transmission from prefrontal cortex in visual selective attention. Nat. Neurosci. 12 (1), 85-91.

Mottaghy, F.M., Krause, B.J., Kemna, L.J., Töpper, R., Tellmann, L., Beu, M., PascualLeone, A., Mueller-Gaertner, H.-W., 2000. Modulation of the neuronal circuitry subserving working memory in healthy human subjects by repetitive transcranial magnetic stimulation. Neurosci. Lett. 280 (3), 167-170.

Nahas, Z. Lomarev, M.P., Roberts, D.R., Shastri, A., Lorberbaum, J.P., Teneback, C., McConnell, K., Vincent, D.J., Li, X., George, M.S., Bohning, D.E., 2001. Unilateral left prefrontal transcranial magnetic stimulation (TMS) produces intensitydependent bilateral effects as measured by interleaved BOLD fMRI. Biol. Psychiatry 50 (9), 712-720.

Nikulin, V.V., Kicić, D., Kähkönen, S., Ilmoniemi, R.J., 2003. Modulation of electroencephalographic responses to transcranial magnetic stimulation: evidence for changes in cortical excitability related to movement. Eur. J. Neurosci. 18 (5), 1206-1212.

Ohnishi, T., Matsuda, H., Imabayashi, E., Okabe, S., Takano, H., Arai, N., Ugawa, Y., 2004a. rCBF changes elicited by rTMS over DLPFC in humans. Suppl. Clin. Neurophysiol. 57, 715-720.

Ohnishi, T., Hayashi, T., Okabe, S., Nonaka, I., Matsuda, H., Iida, H., Imabayashi, E., Watabe, H., Miyake, Y., Ogawa, M., Teramoto, N., Ohta, Y., Ejima, N., Sawada, T., Ugawa, Y., 2004b. Endogenous dopamine release induced by repetitive transcranial magnetic stimulation over the primary motor cortex: an [11C]raclopride positron emission tomography study in anesthetized macaque monkeys. Biol. Psychiatry 55 (5), 484-489.

Okamura, H., Jing, H., Takigawa, M., 2001. EEG modification induced by repetitive transcranial magnetic stimulation. J. Clin. Neurophysiol. 18 (4), 318-325.

Oliviero, A., Strensa, L.H., Di Lazzaro, V., Tonali, P.A., Brown, P., 2003. Persistent effects of high frequency repetitive TMS on the coupling between motor areas in the human. Exp. Brain Res. 149 (1), 107-113.

Ortu, E., Ruge, D., Deriu, F., Rothwell, J.C., 2009. Theta Burst Stimulation over the human primary motor cortex modulates neural processes involved in movement preparation. Clin. Neurophysiol. 120 (6), 1195-1203.

O'Shea, J., Johansen-Berg, Heidi, Trief, D., Göbel, S., Rushworth, M.F.S., 2007. Functionally specific reorganization in human premotor cortex. Neuron 54 (3), 479-490.

Pascual-Leone, A., Walsh, V., Rothwell, J.C., 2000. Transcranial magnetic stimulation in cognitive neuroscience-virtual lesion, chronometry, and functional connectivity. Curr. Opin. Neurobiol. 10 (2), 232-237.

Pasley, B.N., Allen, E.A., Freeman, R.D., 2009. State-dependent variability of neuronal responses to transcranial magnetic stimulation of the visual cortex. Neuron 62 (2), 291-303.

Paus, T., 2005. Inferring causality in brain images: a perturbation approach. Philos. Trans. R. Soc. Lond. B Biol. Sci. 360 (1457), 1109-1114.

Paus, T., Jech, R., Thompson, C.J., Comeau, R., Peters, T., Evans, A.C., 1997. Transcranial magnetic stimulation during positron emission tomography: a new method for studying connectivity of the human cerebral cortex. J. Neurosci. 17 (9), 3178-3184

Paus, T., Jech, R., Thompson, C.J., Comeau, R., Peters, T., Evans, A.C., 1998. Dosedependent reduction of cerebral blood flow during rapid-rate transcranial magnetic stimulation of the human sensorimotor cortex. J. Neurophysiol. 79 (2), 1102-1107.

Paus, T., Sipila, P.K., Strafella, A.P., 2001a. Synchronization of neuronal activity in the human primary motor cortex by transcranial magnetic stimulation: an EEG study. J. Neurophysiol. 86 (4), 1983-1990.

Paus, T., Castro-Alamancos, M.A., Petrides, M., 2001b. Cortico-cortical connectivity of the human mid-dorsolateral frontal cortex and its modulation by repetitive transcranial magnetic stimulation. Eur. J. Neurosci. 14 (8), 1405-1411. 
Pell, G.S., Roth, Y., Zangen, A., 2011. Modulation of cortical excitability induced by repetitive transcranial magnetic stimulation: Influence of timing and geometrical parameters and underlying mechanisms. Prog. Neurobiol. 93, 59-98.

Pleger, B., Blankenburg, F., Bestmann, S., Ruff, C.C., Wiech, K., Stephan, K.E., Friston, K.J., Dolan, R.J., 2006. Repetitive transcranial magnetic stimulation-induced changes in sensorimotor coupling parallel improvements of somatosensation in humans. J. Neurosci. 26 (7), 1945-1952.

Plewnia, C., Rilk, A.J., Soekadar, S.R., Arfeller, C., Huber, H.S., Sauseng, P., Hummel, F., Gerloff, C., 2008. Enhancement of long-range EEG coherence by synchronous bifocal transcranial magnetic stimulation. Eur. J. Neurosci. 27 (6), 1577-1583.

Poreisz, C., Antal, A., Boros, K., Brepohl, N., Csifcsák, G., Paulus, W., 2008. Attenuation of N2 amplitude of laser-evoked potentials by theta burst stimulation of primary somatosensory cortex. Exp. Brain Res. 185 (4), 611-621.

Radman, T., Ramos, R.L., Brumberg, J.C., Bikson, M., 2009. Role of cortical cell type and morphology in sub- and suprathreshold uniform electric field stimulation. Brain Stimul. 2 (4), 215-228.

Ragert, P., Becker, M., Tegenthoff, M., Pleger, B., Dinse, H.R., 2004. Sustained increase of somatosensory cortex excitability by $5 \mathrm{~Hz}$ repetitive transcranial magnetic stimulation studied by paired median nerve stimulation in humans. Neurosci. Lett. 356 (2), 91-94.

Raij, T., Karhu, Jari, Kicić, D., Lioumis, P., Julkunen, P., Lin, F.-H., Ahveninen, J., Ilmoniemi, R.J., Mäkelä, J.P., Hämäläinen, M., Rosen, B.R., Belliveau, J.W., 2008. Parallel input makes the brain run faster. Neuroimage 40 (4), 1792-1797.

Reis, J., Robertson, E., Krakauer, J.W., Rothwell, John, C., Marshall, L., Gerloff, C., Wasermann, E., Pascual-Leone, A., Hummel, F., Celnik, P.A., Classen, J., Floel, A., Ziemann, U., Paulus, W., Siebner, H.R., Born, J., Cohen, L.G., 2008. Consensus: "Can tDCS and TMS enhance motor learning and memory formation? Brain Stimul. 1 (4), 363-369.

Restuccia, D., Ulivelli, M., De Capua, A., Bartalini, S., Rossi, S., 2007. Modulation of high-frequency $(600 \mathrm{~Hz})$ somatosensory-evoked potentials after rTMS of the primary sensory cortex. Eur. J. Neurosci. 26 (8), 2349-2358.

Ridding, M.C., Rothwell, J.C., 2007. Is there a future for therapeutic use of transcranial magnetic stimulation? Nat. Rev. Neurosci. 8 (7), 559-567.

Ridding, M.C., Ziemann, U., 2010. Determinants of the induction of cortical plasticity by non-invasive brain stimulation inn healthy subjects. J. Physiol. 13, 22912304.

Ritter, P., Villringer, A., 2006. Simultaneous EEG-fMRI. Neurosci. Biobehav. Rev. 30 (6), 823-838.

Rudiak, D., Marg, E., 1994. Finding the depth of magnetic brain stimulation: a reevaluation. Electroencephalogr. Clin. Neurophysiol. 93 (5), 358-371.

Rollnik, J.D., Schroeder, C., Rodriguez-Fornells, A., Kurzbuch, A.R., Däuper, J., Moeller, J., Münte, T.F., 2004. Functional lesions and human action monitoring: combining repetitive transcranial magnetic stimulation and event-related brain potentials. Clin. Neurophysiol. 115 (1), 145-153.

Rosanova, M., Casali, A.G., Bellina, V., Resta, F., Mariotti, M., Massimini, M., 2009. Natural frequencies of human corticothalamic circuits. J. Neurosci. 29 (24), 7679-7685.

Rossi, S., Pasqualetti, P., Rossini, P.M., Feige, B., Ulivelli, M., Glocker, F.X., Battistini, N., Lucking, C.H., Kristeva-Feige, R., 2000. Effects of repetitive transcranial magnetic stimulation on movement-related cortical activity in humans. Cereb. Cortex 10 (8), 802-808.

Rounis, E., Lee, L., Siebner, H.R., Rowe, J.B., Friston, K.J., Rothwell, J.C., Frackowiak, S.J., 2005. Frequency specific changes in regional cerebral blood flow and motor system connectivity following rTMS to the primary motor cortex. Neuroimage 26 (1), 164-176.

Ruff, C.C., Blankenburg, F., Bjoertomt, O., Bestmann, S., Freeman, E., Haynes, J.-D., Rees, G., Josephs, O., Deichmann, R., Driver, J., 2006. Concurrent TMS-fMRI and psychophysics reveal frontal influences on human retinotopic visual cortex. Curr. Biol. 16 (15), 1479-1488.

Ruff, C.C., Bestmann, S., Blankenburg, F., Bjoertomt, O., Josephs, O., Weiskopf, N., Deichmann, R., Driver, J., 2008. Distinct causal influences of parietal versus frontal areas on human visual cortex: evidence from concurrent TMS-fMRI. Cereb. Cortex 18 (4), 817-827.

Ruff, C.C., Driver, J., Bestmann, S., 2009a. Combining TMS and fMRI: from "virtual lesions" to functional-network accounts of cognition. Cortex 45 (9), 1043-1049.

Ruff, C.C., Blankenburg, F., Bjoertomt, O., Bestmann, S., Weiskopf, N., Driver, J., 2009b. Hemispheric differences in frontal and parietal influences on human occipital cortex: direct confirmation with concurrent TMS-fMRI. J. Cogn. Neurosci. 21 (6), 1146-1161.

Sack, A.T., 2006. Transcranial magnetic stimulation, causal structure-function mapping and networks of functional relevance. Curr. Opin. Neurobiol. 16 (5), 593-599.

Sack, A.T., 2010. Does TMS need functional imaging? Cortex 46 (1), 131-133.

Sack, A.T., Linden, D.E.J., 2003. Combining transcranial magnetic stimulation and functional imaging in cognitive brain research: possibilities and limitations. Brain Res. Rev. 43 (1), 41-56.

Sack, A.T., Camprodon, J.A., Pascual-Leone, A., Goebel, R., 2005. The dynamics of interhemispheric compensatory processes in mental imagery. Science 308 (5722), 702-704.

Sack, A.T., Kohler, A., Bestmann, S., Linden, D.E.J., Dechent, P., Goebel, R., Baudewig, J., 2007. Imaging the brain activity changes underlying impaired visuospatial judgments: simultaneous FMRI TMS, and behavioral studies. Cereb. Cortex 17 (12), 2841-2852.

Satow, T., Mima, T., Yamamoto, J., Oga, T., Begum, T., Aso, T., Hashimoto, N., Rothwell, J.C., Shibasaki, H., 2003. Short-lasting impairment of tactile perception by $0.9 \mathrm{~Hz}-\mathrm{rTMS}$ of the sensorimotor cortex. Neurology 60 (6), 1045-1047.
Sağlam, M., Matsunaga, K., Murayama, N., Hayashida, Y., Huang, Y.-Z., Nakanishi, R., 2008. Parallel inhibition of cortico-muscular synchronization and cortico-spinal excitability by theta burst TMS in humans. Clin. Neurophysiol. 119 (12), 28292838.

Schindler, K., Nyffeler, T., Wiest, Roland, Hauf, M., Mathis, J., Hess, C.W., Müri, R., 2008. Theta burst transcranial magnetic stimulation is associated with in creased EEG synchronization in the stimulated relative to unstimulated cerebral hemisphere. Neurosci. Lett. 436 (1), 31-34.

Schutter, D.J., van Honk, J., d'Alfonso, A.A., Postma, A., de Haan, E.H., 2001. Effects of slow rTMS at the right dorsolateral prefrontal cortex on EEG asymmetry and mood. Neuroreport 12 (3), 445-447.

Schutter, D.J., van Honk, J., 2003. Reductions in CI amplitude after repetitive transcranial magnetic stimulation (rTMS) over the striate cortex. Brain Res. Cogn. Brain Res. 16 (3), 488-491.

Schutter, D.J., van Honk, J., d'Alfonso, A.A., Peper, J.S., Panksepp, J., 2003. High frequency repetitive transcranial magnetic over the medial cerebellum induces a shift in the prefrontal electroencephalography gamma spectrum: a pilot study in humans. Neurosci. Lett. 336, 73-76.

Schutter, D.J., van Honk, J., 2006. An electrophysiological link between the cerebellum, cognition and emotion: frontal theta EEG activity to single-pulse cerebellar TMS. Neuroimage 33 (4), 1227-1231.

Schürmann, M., Nikouline, V.V., Soljanlahti, S., Ollikainen, M., Basar, E., Ilmoniemi, R.J., 2001. EEG responses to combined somatosensory and transcranial magnetic stimulation. Clin. Neurophysiol. 112 (1), 19-24.

Seyal, M., Browne, J.K., Masuoka, L.K., Gabor, A.J., 1993. Enhancement of the amplitude of somatosensory evoked potentials following magnetic pulse stimulation of the human brain. Electroencephalogr. Clin. Neurophysiol. 88 (1), 20 27.

Sibon, I., Strafella, A.P., Gravel, P., Ko, J.H., Booij, L., Soucy, J.P., Leyton, M., Diksic, M., Benkelfat, C., 2007. Acute prefrontal cortex TMS in healthy volunteers: effects on brain ${ }^{11} \mathrm{C}$-alphaMtrp trapping. Neuroimage 34 (4), 1658-1664.

Siebner, H.R., Willoch, F., Peller, M., Auer, C., Boecker, H., Conrad, B., Bartenstein, P., 1998. Imaging brain activation induced by long trains of repetitive transcranial magnetic stimulation. Neuroreport 9 (5), 943-948.

Siebner, H.R., Peller, M., Willoch, F., Minoshima, S., Boecker, H., Auer, C., Drzezga, A., Conrad, B., Bartenstein, P., 2000. Lasting cortical activation after repetitive TMS of the motor cortex: a glucose metabolic study. Neurology 54 (4), 956-963.

Siebner, H.R., Peller, M., Bartenstein, P., Willoch, F., Rossmeier, C., Schwaiger, M., Conrad, B., 2001a. Activation of frontal premotor areas during suprathreshold transcranial magnetic stimulation of the left primary sensorimotor cortex: a glucose metabolic PET study. Hum. Brain Mapp. 12, 157-167.

Siebner, H.R., Takano, B., Peinemann, A., Schwaiger, M., Conrad, B., Drzezga, A., 2001b. Continuous transcranial magnetic stimulation during positron emission tomography: a suitable tool for imaging regional excitability of the human cortex. Neuroimage 14, 883-890.

Siebner, H.R., Bergmann, T.O., Bestmann, S., Massimini, M., Johansen-Berg, Heidi, Mochizuki, H., et al., 2009. Consensus paper: combining transcranial stimulation with neuroimaging. Brain Stimul. 2 (2), 58-80.

Speer, A.M., Willis, M.W., Herscovitch, P., Daube-Witherspoon, M., Shelton, J.R., Benson, B.E., Post, R.M., Wassermann, E.M., 2003a. Intensity-dependent regional cerebral blood flow during $1-\mathrm{Hz}$ repetitive transcranial magnetic stimulation (rTMS) in healthy volunteers studied with $\mathrm{H}_{2}{ }^{15} \mathrm{O}$ positron emission tomography: I. Effects of primary motor cortex rTMS. Biol. Psychiatry 54, 818-825.

Speer, A.M., Willis, M.W., Herscovitch, P., Daube-Witherspoon, M., Shelton, J.R., Benson, B.E., Post, R.M., Wassermann, E.M., 2003b. Intensity-dependent regional cerebral blood flow during $1-\mathrm{Hz}$ repetitive transcranial magnetic stimulation (rTMS) in healthy volunteers studied with $\mathrm{H}_{2}{ }^{15} \mathrm{O}$ positron emission tomography: II. Effects of prefrontal cortex rTMS. Biol. Psychiatry 54 (8), 826-832.

Stagg, C.J., Wylezinska, M., Matthews, P.M., Johansen-Berg, H., Jezzard, P., Rothwell, J.C., Bestmann, S., 2009. Neurochemical effects of theta burst stimulation as assessed by magnetic resonance spectroscopy. J. Neurophysiol. 101 (6), 28722877.

Strafella, A.P., Paus, T., Barrett, J., Dagher, A., 2001. Repetitive transcranial magnetic stimulation of the human prefrontal cortex induces dopamine release in the caudate nucleus. J. Neurosci. 21 (15), RC157.

Strafella, A.P., Paus, T., Fraraccio, M., Dagher, A., 2003. Striatal dopamine release induced by repetitive transcranial magnetic stimulation of the human motor cortex. Brain 126 (Pt 12), 2609-2615.

Strens, L.H.A., Oliviero, A., Bloem, B.R., Gerschlager, W., Rothwell, J.C., Brown, P., 2002. The effects of subthreshold $1 \mathrm{~Hz}$ repetitive TMS on cortico-cortical and interhemispheric coherence. Clin. Neurophysiol. 113 (8), 1279-1285.

Taylor, P.C.J., Nobre, A.C., Rushworth, M.F.S., 2007a. FEF TMS affects visual cortical activity. Cereb. Cortex 17 (2), 391-399.

Taylor, P.C.J., Nobre, A.C., Rushworth, M.F.S., 2007b. Subsecond changes in top down control exerted by human medial frontal cortex during conflict and action selection: a combined transcranial magnetic stimulation electroencephalography study. J. Neurosci. 27 (42), 11343-11353.

Taylor, P.C.J., Walsh, V., Eimer, M., 2008. Combining TMS and EEG to study cognitive function and cortico-cortico interactions. Behav. Brain Res. 191 (2), 141-147.

Thickbroom, G.W., 2007. Transcranial magnetic stimulation and synaptic plasticity: Experimental framework and human models. Exp. Brain Res. 180, 583-593.

Thut, G., Northoff, G., Ives, J.R., Kamitani, A., Pfennig, A., Kampmann, F., Schomer D.L., Pascual-Leone, A., 2003a. Effects of single-pulse transcranial magnetic stimulation (TMS) on functional brain activity: a combined event-related TMS and evoked potential study. Clin. Neurophysiol. 114 (11), 2071-2080. 
Thut, G., Theoret, H., Pfennig, A., Ives, J.R., Kampmann, F., Northoff, G., PascualLeone, A., 2003b. Differential effects of low-frequency rTMS at the occipital pole on visual-induced alpha desynchronization and visual-evoked potentials. Neuroimage 18 (2), 334-347.

Thut, G., Miniussi, C., 2009. New insights into rhythmic brain activity from TMS-EEG studies. Trends Cogn. Sci. 13 (4), 182-189.

Thut, G., Pascual-Leone, A., 2010. A review of combined TMS-EEG studies to characterize lasting effects of repetitive TMS and assess their usefulness in cognitive and clinical neuroscience. Brain Topogr. 22 (4) 219-232.

Trippe, J., Mix, A., Aydin-Abidin, S., Funke, K., Benali, A., 2009. Theta burst and conventional low-frequency rTMS differentially affect GABAergic neurotransmission in the rat cortex. Exp. Brain Res. 199, 411-421.

Tsuji, T., Rothwell, J.C., 2002. Long lasting effects of rTMS and associated peripheral sensory input on MEPs SEPs and transcortical reflex excitability in humans. J. Physiol. 540 (1), 367-376.

Urushihara, R., Murase, N., Rothwell, J.C., Harada, M., Hosono, Y., Asanuma, K. Shimazu, H., Nakamura, K., Chikahisa, S., Kitaoka, K., Sei, H., Morita, Y., Kaji, R., 2006. Effect of repetitive transcranial magnetic stimulation applied over the premotor cortex on somatosensory-evoked potentials and regional cerebral blood flow. Neuroimage 31 (2), 699-709.

Valero-Cabré, A., Payne, B.R., Rushmore, J., Lomber, S.G., Pascual-Leone, A., 2005. Impact of repetitive transcranial magnetic stimulation of the parietal cortex on metabolic brain activity: a 14C-2DG tracing study in the cat. Exp. Brain Res. 163 (1), 1-12.

Valero-Cabré, A., Payne, B.R., Pascual-Leone, A., 2007. Opposite impact on 14C-2 deoxyglucose brain metabolism following patterns of high and low frequency repetitive transcranial magnetic stimulation in the posterior parietal cortex. Exp. Brain Res. 176 (4), 603-615.

van der Werf, Y.D., Paus, T., 2006. The neural response to transcranial magnetic stimulation of the human motor cortex, intracortical and cortico-cortical contributions. Exp. Brain Res. 175 (2), 231-245.

Wagner, T., Valero-Cabre, A., Pascual-Leone, A., 2007. Noninvasive human brain stimulation. Annu. Rev. Biomed. Eng. 9, 527-565.

Wagner, T., Rushmore, J., Eden, U., Valero-Cabre, A., 2009. Biophysical foundations underlying TMS: setting the stage for an effective use of neurostimulation in the cognitive neurosciences. Cortex 45 (9), 1025-1034.

Walsh, V., Ellisona, Battelli, L., Cowey, A., 1998. Task-specific impairments and enhancements induced by magnetic stimulation of human visual area V5. Proc. Biol. Sci. 265 (1395), 537-543.

Walsh, V., Cowey, A., 2000. Transcranial magnetic stimulation and cognitive neuroscience. Nat. Rev. Neurosci. 1 (1), 73-79.

Wassermann, E.M., Epstein, C.M., Ziemann, U., Walsh, V., Paus, T., Lisanby, S.H. (Eds.), 2008. The Oxford Handbook of Transcranial Stimulation. Oxford University Press, Oxford.

Wolters, A., Schmidt, A., Schramm, A., Zeller, D., Naumann, M., Kunesch, E., Benecke, R., Reiners, K., Classen, J., 2005. Timing-dependent plasticity in human primary somatosensory cortex. J. Physiol. 565 (Pt 3), 1039-1052.

Yoo, W.-K., You, S.H., Ko, M.-H., Tae Kim, S., Park, C.-H., Park, J.-W., Ohn, S.H., Hallett, M., Kim, Y.-H., 2008. High frequency rTMS modulation of the sensorimotor networks: behavioral changes and fMRI correlates. Neuroimage 39 (4), 1886-1895.

Zanon, M., Busan, P., Monti, F., Pizzolato, G., Battaglini, P.P., 2010. Cortical connections between dorsal and ventral visual streams in humans: evidence by TMS/ EEG co-registration. Brain Topogr. 22 (4), 307-317. 